JOURNAL OF

SYMPLECTIC GEOMETRY

Volume 1, Number 2, 269-320, 2002

\title{
Strict Quantization of Solvable Symmetric Spaces
}

\author{
PierRe BieliaVsky
}

To the memory of Moshé Flato

This work is a contribution to the area of Strict Quantization (in the sense of Rieffel) in the presence of curvature and nonAbelian group actions. More precisely, we use geometry to obtain explicit oscillatory integral formulae for strongly invariant strict deformation quantizations of a class of solvable symplectic symmetric spaces. Each of these quantizations gives rise to a field of (pre)- $C^{\star}$-algebras whose fibers are function algebras which are closed under the deformed product. The symmetry group of the symmetric space acts on each fiber by $C^{\star}$-algebra automorphisms.

\section{Introduction.}

Weyl's method for quantizing a free particle in $\mathbb{R}^{n}$ consists in a correspondence between classical observables (i.e., functions on the phase space $\left.\mathbb{R}^{2 n}=T^{\star}\left(\mathbb{R}^{n}\right)\right)$ and linear operators acting on the Hilbert space of square integrable functions on the configuration space $\mathbb{R}^{n}$. By reading the operator composition product at the level of functions via Weyl's correspondence, one gets a non-commutative associative product on the classical observables called Weyl's product. This new product appears as a deformation with one parameter, namely Planck's "constant" $\hbar$, of the usual commutative pointwize multiplication of functions in the direction of the classical Poisson bracket. This is the starting point of Formal Deformation Quantization (FDQ) theory (or star products), as introduced by Bayen, Flato, Fronsdal, Lichnerowicz and Sternheimer in the late seventies [2]. In FDQ, Quantum Mechanics is formulated in a classical framework as the space of formal power series in $\hbar$ with classical observables as coefficients. No reference to a particular Hilbert space representation is made.

An important feature of Weyl's product is that it is "strict", meaning that in a suitable functional framework, the product of two functions is again a function, rather than a formal power series in $\hbar$, as in star product theory. For instance, the Schwartz space on $\mathbb{R}^{2 n}$ is closed under Weyl's product. Originally formulated in the framework of $C^{\star}$-algebras, the notion (theory) 
of Strict Deformation Quantization (SDQ) was introduced by M. A. Rieffel in 1989 [22] (see also [16]), and has been extensively developed in various directions since then. For example, SDQ provides a very elegant and simple description of Quantum Tori by generalizing Weyl's quantization of $\mathbb{R}^{2 n}$ to Poisson manifolds whose Poisson structure comes from an action of $\mathbb{R}^{d}[21]$. In the latter, invariance of Weyl's product under translations plays a crucial role.

There is a very simple oscillatory integral formula for Weyl's deformed product (see formula (1) in the next section). Such an oscillatory integral formula is not only convenient when studying the functional analysis and strictness of the quantization, it also indicates a possible approach to the study of the geometry of the quantization.

On a symplectic manifold $M$, it is natural to express a given (continuous) multiplication, $\star$, on functions via a kernel formula of the type:

$$
u \star v(x)=\int_{M \times M} K(x, ., .) u \otimes v,
$$

where $u$ and $v$ are two functions on $M$, where $x$ is a point in $M$, and where $K$ is the three-point kernel defining the multiplication $\star$. The integration is with respect to the product Liouville measure on $M \times M$.

In the so-called WKB-quantization program (see [25]) initiated independently by Karasev, Weinstein and Zakrzewski, one considers kernels of the form:

$$
K=a_{\hbar} e^{\frac{i}{\hbar} S}
$$

Here $S$ is a real-valued smooth function on $M \times M \times M$ called the phase and $a_{\hbar}$, called the amplitude, is usually a power series in $\hbar$. It is defined in such a way that the product $\star$ would be, at least formally, associative. It should also constitute a one-parameter $(\hbar)$ deformation of the usual pointwize product in the direction of the symplectic Poisson bracket. Briefly, a WKB-quantization is defined by an oscillatory integral product formula.

When one imposes compatibility with some geometric structure given on the symplectic manifold, for instance invariance of the quantization under the action of the group of affine transformations of a given symplectic connection, $S$ and $a_{\hbar}$ become extremely constrained and carry non-trivial geometric information. In the simplest example of Weyl's quantization of $M=\mathbb{R}^{2 n}$, the amplitude function is identically equal to 1 while the phase $S(x, y, z)$ is proportional to the symplectic area of the Euclidean triangle $\Delta x y z$ whose vertices are points $x, y$ and $z[12,25]$. In particular, in this 
case the largest group of diffeomorphisms of $\mathbb{R}^{2 n}$ preserving the quantization is $S p(n, \mathbb{R}) \times \mathbb{R}^{2 n}$, that is, the group of symplectic displacements with respect to the flat connection on $\mathbb{R}^{2 n}$.

In this work, we study WKB-quantizations of a class of curved affine symplectic manifolds. More precisely, a symplectic symmetric space is a triple $(M, \omega, \nabla)$ where $(M, \omega)$ is a symplectic manifold and where $\nabla$ is a torsion-free affine connection on $M$ such that $\nabla \omega=0$ and such that at every point $x \in M$, the local geodesic symmetry, $s_{x}$, extends globally to $M$ as an affine symplectic transformation. In this case the group $G(M)$ of transformations of $M$ generated by the symmetries $\left\{s_{x}\right\}$ completely determines the connection (see Section 2). By a WKB-quantization of a symplectic symmetric space, we essentially mean a WKB-quantization of $(M, \omega)$ with kernel $K=a_{\hbar} e^{\frac{i}{\hbar} S}$ as above such that

(i) the amplitude $a_{\hbar}$ and the phase $S$ are invariant under the group $G(M)$;

(ii) for all value of $\hbar \in[0, \infty)$, there exists a function algebra $\mathcal{E}_{\hbar} \subset \operatorname{Fun}(M)$ stable under the deformed multiplication and containing the space of smooth compactly supported functions. (We denote by $\operatorname{Fun}(M)$ the space of complex valued functions on $M$ ).

For a precise definition see Definition 1.5.

One interest of such an invariant quantization is that, when well behaved, it leads to deformations of quotient spaces $\Gamma \backslash M$ where $\Gamma \subset G(M)$ (see Section 8). However, this last question will not be investigated in the present article.

WKB-quantization of symplectic symmetric spaces has already been investigated by A. Weinstein. In [25], assuming the existence of a WKBquantization of a symmetric symplectic space which is invariant under the group of automorphisms, Weinstein gives a beautiful geometric description of the phase $S(x, y, z)$ in terms of the symplectic area of a geodesic triangle admitting points $x, y$ and $z$ as midpoints of its edges. Even for symmetric spaces, the problems of finding the amplitude $a_{\hbar}$ as well as that of giving a suitable functional framework where the WKB-quantization would yield topological function algebras are still wide open. Nevertheless, a geometrical study combined with techniques coming from Star Representation theory $[1,10]$ (representation theory of Lie groups in the framework of deformation quantization) seem to suggest a way to attend these questions. At least for solvable symmetric spaces, one can give a quite satisfying answer. This is what is done in this paper. The symplectic spaces considered here are symplectomorphic to $\mathbb{R}^{2 n}$ endowed with its standard symplectic structure. From 
the topological point of view the situation is therefore trivial. In contrast, the geometry is not. Indeed, each of these spaces is endowed with a curved symplectic affine connection whose symplectic affine transformations are not linear. In particular, Weyl's product is not preserved under such canonical transformations. In our case, the automorphism group $G(M)$ is a solvable Lie group. Our situation therefore differs from the Kählerian symmetric case whose study was initiated by Berezin [3] (see Section 8).

The present paper is organized as follows.

\section{Weyl's Quantization revisited}

We introduce a geometric setting in which associativity of Weyl's product is interpreted in elementary geometric terms. We propose a convenient framework for WKB-quantization (Definition 1.5) inspired by Rieffel's definition of strict deformation quantization.

\section{General facts about symplectic symmetric spaces}

For the convenience of the reader, we recall some relevant definitions and results on symplectic symmetric spaces which can be found in [5].

\section{Phase functions on symplectic symmetric spaces}

We adapt the geometric setting introduced in Section 1 to the case of symplectic symmetric spaces. We indicate how Weinstein's phase function [25] appears in this picture.

\section{Elementary solvable symplectic symmetric spaces}

We define the class of symplectic symmetric spaces that will be considered in this work. Those are such that the action of the holonomy algebra at a point $o$ has an isotropic range in the symplectic tangent space at $o$. The symmetry group of such a symplectic symmetric space is a solvable Lie group. Among solvable symplectic symmetric spaces, these spaces are structurally of primary importance (see Section 4 and [7]). We call them elementary solvable symplectic symmetric spaces. We end this section by giving explicit formulae for the invariant phase functions $S$ on these spaces as well as an embedding property (Proposition 4.7) which will be useful when studying the functional analysis attached to the integral kernel $K=a_{\hbar} e^{\frac{i}{\hbar} S}$. 


\section{Obtaining the oscillating kernel via star representation the- oretical methods}

On a symplectic symmetric space as considered in Section 4, one has a canonical Darboux chart. In this Darboux chart, the formal Moyal star product turns out to be covariant (in Arnal's sense) under the action of some central extension of the transvection algebra. Using the cocycle defining the associated star representation, we introduce some kind of integral Fourier operator on the quantum algebra which intertwines the usual commutative product with an $\hbar$-dependent commutative product for which the extended transvection algebra acts by derivations. The "commutative manifold" underlying the latter product carries therefore a strongly invariant deformation quantization. This space turns out to be equivariantly isomorphic to the symmetric space at hand.

\section{WKB-Quantization}

We determine explicitly the amplitude functions $a_{\hbar}$ that yield invariant WKB-quantizations of our solvable symplectic symmetric spaces via their associated kernels $K=a_{\hbar} e^{\frac{i}{\hbar} S}$. Combined with Section 5, this yields explicit oscillatory integral formulae for strongly invariant strict deformation quantizations (Theorem 6.13). We define deformed function algebras i.e., we exhibit spaces of functions on the symmetric space which are stable under the oscillatory integral deformed product.

\section{Topological algebras}

We define symmetry invariant $C^{\star}$-norms on the above mentioned function algebras.

\section{Remarks for further developments}

The present work gives us a hope for attending "quantum Anosov property" on compact solv-manifolds. Also, we believe it could allow to attend "quantum surfaces" ([20], [17]) in an explicit and quite elementary way.

Acknowledgments. The author would like to express his gratitude to Alan Weinstein for many enlightening discussions as well as an invitation in January of 2000 at the Mathematics Department of UC Berkeley. Considerable improvements to this paper were made there. He also would like to thank Marc Rieffel for communicating his work on deformation quantization for actions of $\mathbb{R}^{d}$ and pointing out the right framework for "convergent" 
deformation quantization. The author is indebted to Mélanie Bertelson for having suggested the important choice of a norm in the proof of Lemma 6.2. At last, the author thanks Jeff Kiralis and Stefan Waldmann for reading the first draft of the manuscript and suggesting improvements to it. This research has been partially supported by the Communauté Française de Belgique, through an Action de Recherche Concertée de la Direction de la Recherche Scientifique.

\section{Weyl's Quantization revisited ${ }^{1}$.}

Weyl's quantization consists in a correspondence between classical observables of a mechanical system, i.e., functions on a phase space and operators on a suitable Hilbert space. More precisely, when the phase space is the symplectic vector space $\left(\mathbb{R}^{2 n}, \omega^{0}\right)$, Weyl's quantization map is given by:

$$
\begin{gathered}
\mathcal{S}\left(\mathbb{R}^{2 n}\right) \stackrel{W_{\hbar}}{\rightarrow} \mathcal{B}\left(L^{2}\left(\mathbb{R}^{n}\right)\right) \\
\left(W_{\hbar}(u) f\right)(q)=\int_{\mathbb{R}^{n} \times \mathbb{R}^{n}} e^{\frac{i}{\hbar}\langle q-\eta, \xi\rangle} u\left(\frac{q+\eta}{2}, \xi\right) f(\eta) d \xi d \eta,
\end{gathered}
$$

where $\mathbb{R}^{2 n}$ is seen as $\mathbb{R}^{n} \times \mathbb{R}^{n}=\{(q, p)\}, d \xi$ (respectively $d \eta$ ) is some suitable normalization of the Haar measure on the Abelian Lie group $\mathbb{R}^{n}$, where $\mathcal{S}\left(\mathbb{R}^{2 n}\right)$ denotes the space of Schwartz's functions on $\mathbb{R}^{2 n}$ and $\mathcal{B}\left(L^{2}\left(\mathbb{R}^{n}\right)\right)$ is the algebra of bounded operators on $L^{2}\left(\mathbb{R}^{n}\right)$. The Weyl product of two Schwartz's functions is formally defined as:

$$
W_{\hbar}\left(u \star_{\hbar}^{0} v\right)=W_{\hbar}(u) \circ W_{\hbar}(v) .
$$

One derives the following integral formula for the Weyl product:

$$
\left(u \star_{\hbar}^{0} v\right)(x)=\frac{1}{\hbar^{2 n}} \int_{\mathbb{R}^{2 n} \times \mathbb{R}^{2 n}} u(y) v(z) e^{-\frac{2 i}{\hbar} S^{0}(x, y, z)} d y d z
$$

where

$$
S^{0}(x, y, z)=\omega^{0}(x, y)+\omega^{0}(y, z)+\omega^{0}(z, x),
$$

and where $d y$ (respectively $d z$ ) is some suitable normalization of the Liouville measure.

\footnotetext{
${ }^{1}$ In this section we will not consider the functional analytical problems involved in existence and well-definedness of our oscillatory integrals, those will be investigated in the next sections. Only the geometric aspects will be discussed in the present section.
} 
Endowed with this product, the Schwartz space $\left(\mathcal{S}\left(\mathbb{R}^{2 n}\right), \star_{\hbar}^{0}\right)$ becomes an associative topological algebra [11]. Its algebra structure extends to $L^{2}\left(\mathbb{R}^{2 n}\right)$.

Interpreting formula (1) as an oscillatory integral with parameter $-\frac{2}{\hbar}$, one can use a stationary phase method to obtain the following asymptotic expansion:

$$
u \star_{\hbar}^{0} v \sim u v+\nu\{u, v\}+\sum_{k=2}^{\infty} \frac{\nu^{k}}{k !} \sum_{\substack{i_{1} \ldots i_{k} \\ j_{1} \ldots j_{k}}} \Omega^{i_{1} j_{1}} \ldots \Omega^{i_{k} j_{k}} \partial_{i_{1} \ldots i_{k}} u . \partial_{j_{1} \ldots j_{k}} v
$$

with $\nu=\frac{\hbar}{2 i}$ and where $\Omega^{i j} \partial_{i} \wedge \partial_{j}=\{$,$\} is the Poisson tensor associated to$ $\omega^{0}$. The RHS of (2) extends by $\mathbb{C}[[\hbar]]$-bilinearity as an associative product to the space of formal power series $C^{\infty}\left(\mathbb{R}^{2 n}\right)[[\hbar]]$. This formal product is called the Moyal star product and will be denoted by $u \star_{\nu}^{M} v$.

Dirac's condition in Quantum Mechanics in this context reads as

$$
\frac{1}{2 \nu}\left(u \star_{\nu}^{M} v-v \star_{\nu}^{M} u\right) \bmod (\nu)=\{u, v\}
$$

The oscillatory integral formula (1) indicates that the triple $\left(\mathbb{R}^{2 n}, d x, S^{0}\right)$ contains all the structure needed to produce an associative non commutative deformation of the usual pointwize product on functions on $\mathbb{R}^{2 n}$. Of course, compatibility between $S^{0}$ and the (Abelian) group structure of $\mathbb{R}^{2 n}$ is certainly crucial. So, a natural question is: given an orientable manifold $M$ endowed with a volume form $\mu$, what are the conditions on a three-point function $S \in C^{\infty}(M \times M \times M, \mathbb{R})$ which would guarantee associativity of the product

$$
u \star v(x)=\int_{M \times M} e^{i S(x, ., .)} u \otimes v \mu \otimes \mu ?
$$

When writing a (continuous) multiplication on functions via a kernel formula of the type:

$$
u \star v(x)=\int_{M \times M} K(x, ., .) u \otimes v
$$

a computation shows that associativity for the multiplication $\star$ is (at least formally) equivalent to the following condition:

$$
\int_{M} K(a, b, t) K(t, c, d) \mu(t)=\int_{M} K(a, \tau, d) K(\tau, b, c) \mu(\tau),
$$


for every quadruple of points $a, b, c, d$ in $M$. Equality (3) obviously holds if one can pass from one integrand to the other using a change a variables $\tau=\varphi(t)$. This motivates

Definition 1.1. Let $(M, \mu)$ be an orientable manifold endowed with a volume form $\mu$. A three-point kernel $K \in C^{\infty}(M \times M \times M)$ is geometrically associative if for every quadruple of points $a, b, c, d$ in $M$ there exists a volume preserving diffeomorphism

$$
\varphi:(M, \mu) \rightarrow(M, \mu)
$$

such that for all $t$ in $M$ :

$$
K(a, b, t) K(t, c, d)=K(a, \varphi(t), d) K(\varphi(t), b, c) .
$$

We will prove in Proposition 1.3 that the following structure leads to a geometrically associative kernel.

Definition 1.2. A Weyl triple is a triple $(M, \mu, S)$ where $(M, \mu)$ is an oriented manifold and $S: M \times M \times M \rightarrow \mathbb{R}$ is a smooth three-point function on $M$ such that:

(i) $S(x, y, z)=S(z, x, y)=-S(y, x, z)$;

(ii) for all $m \in M$, one has:

$$
S(x, y, z)=S(x, y, m)+S(y, z, m)+S(z, x, m) \quad \forall x, y, z \in M
$$

(iii) for all $x \in M$, there exists a $\mu$-preserving diffeomorphism $s_{x}: M \rightarrow M$ such that:

$$
S(x, y, z)=-S\left(x, s_{x}(y), z\right) \quad \forall y, z \in M
$$

Property (i) in Definition 1.2 naturally leads us to adopt the following "oriented graph" type notation for $S$ :

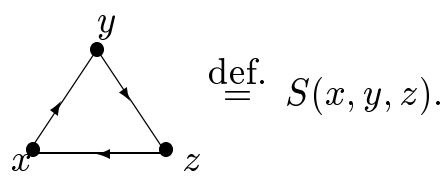

A change of orientation in such an "oriented triangle" leads to a change of sign of its value. However, the value represented by such a "triangle" does 
not depend on the way it "stands", only the data of the vertices and the orientation of the edges matters.

Now, consider a Weyl triple $(M, \mu, S)$, and let $A$ be some (topological) associative algebra. And, for compactly supported functions $u$ and $v \in$ $C_{c}^{\infty}(M, A)$, consider the following "product":

$$
u \star v(x)=\int_{M \times M} u(y) v(z) e^{i S(x, y, z)} \mu(y) \mu(z) .
$$

With the above notation for $S$, associativity for $\star$ now formally reads as follows:

$$
\begin{aligned}
& u \star(v \star w)(a)=\int \exp i(\overbrace{d}^{a}) \int \exp i(t) v(c) w(d) \\
& =\int \exp i(\overbrace{\tau}^{a}) \int \exp i\left(\sigma_{c}\right) u(b) v(c) w(d)=(u \star v) \star w(a),
\end{aligned}
$$

the $\mu$-integration being taken over variables $b, c, d, t$ and $\tau$. This leads, for $K=e^{i S}$, to an equality between two "distribution valued functions" on $M \times M \times M \times M$ (cf. formula (3)): for every quadruple of points $a, b, c, d$ in $M$ associativity for $\star$ reads

(4)

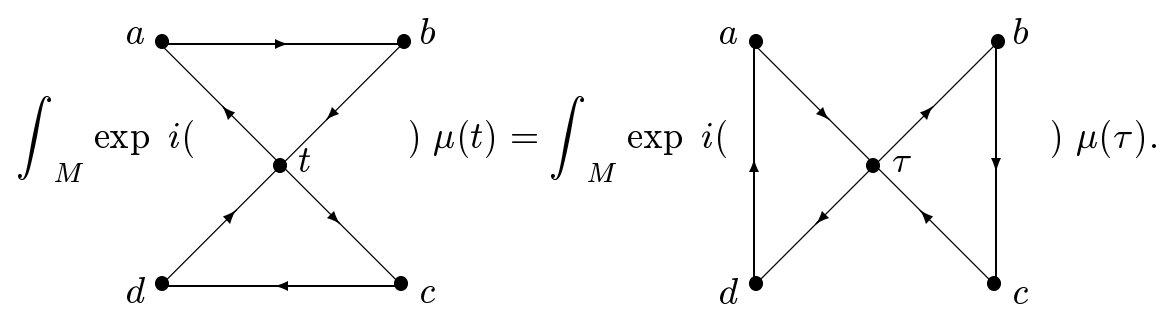

In the above formula, the diagram in the argument of the exponential in the LHS (respectively the RHS) stands for $S(a, b, t)+S(t, c, d)$ (respectively $S(a, d, \tau)+S(\tau, b, c))$. 
Proposition 1.3. Let $(M, \mu, S)$ be a Weyl triple. Then, the associated three-point kernel $K=e^{i S}$ is geometrically associative.

Proof. Fix four points $a, b, c, d$. Regarding Definition 1.1 and formula (4), one needs to construct our volume preserving diffeomorphism $\varphi:(M, \mu) \rightarrow$ $(M, \mu)$ in such a way that for all $t$,
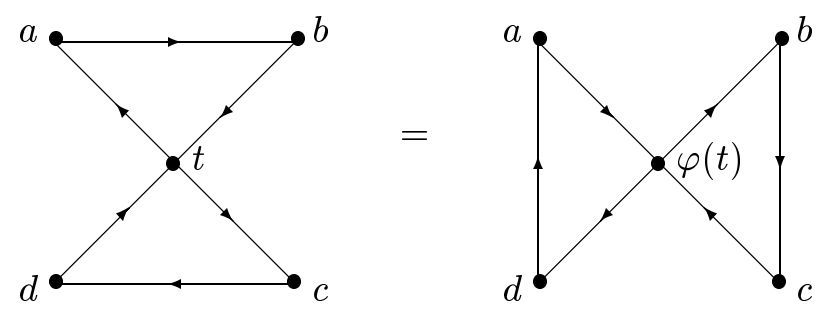

We first observe that the data of four points $a, b, c, d$ determines what we call an " $S$-barycenter", that is a point $g=g(a, b, c, d)$ such that
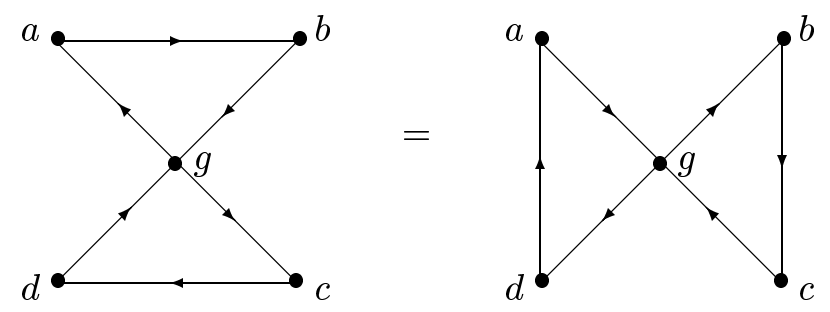

Indeed, since
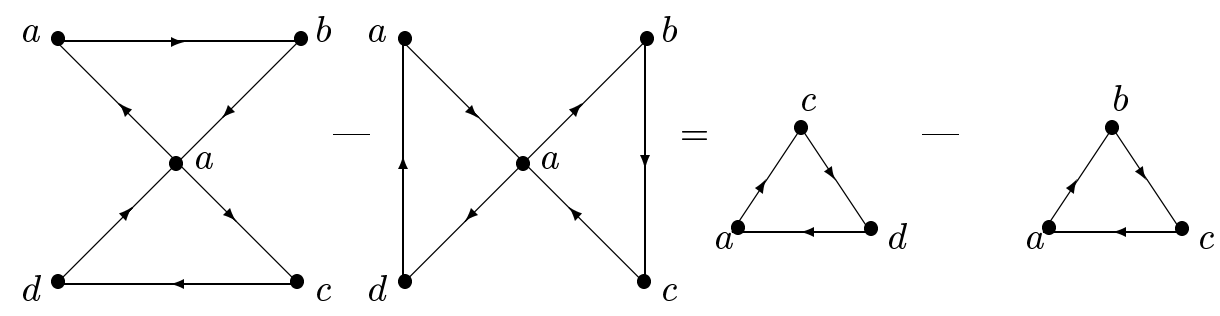

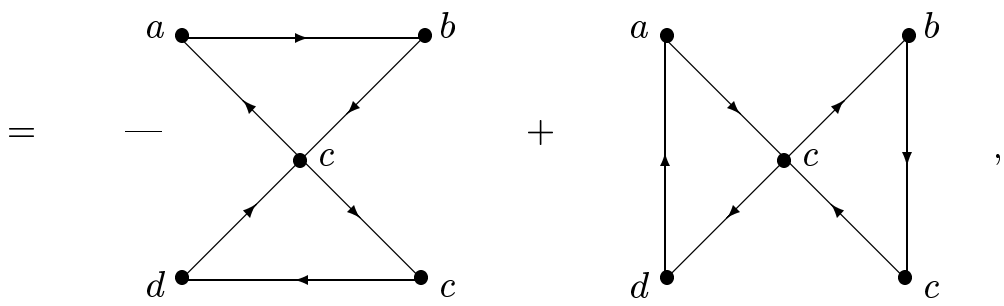

any continuous path joining $a$ to $c$ contains such a point $g$.

Now, we fix once for all such an $S$-barycenter $g$ for $\{a, b, c, d\}$ and we adopt the following notation. For all $x$ and $y$ in $M$, the value of $S(g, x, y)$ is denoted by a "thickened arrow":

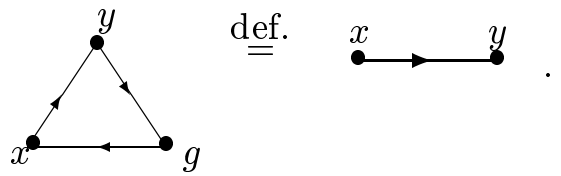

Again, a change of orientation in such an arrow changes the sign of its value. Also, property (iii) of a Weyl triple (Definition 1.2) which reads
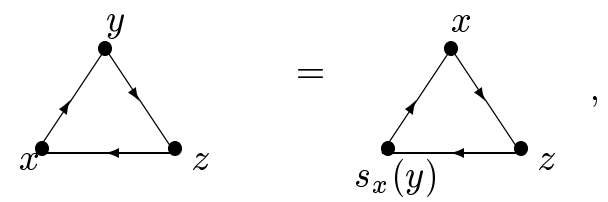

implies

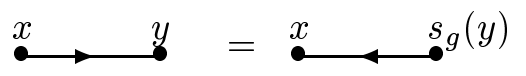


for all $x$ and $y$ in $M$. While, from property (ii) (Definition 1.2 with $m=g$ ), one gets

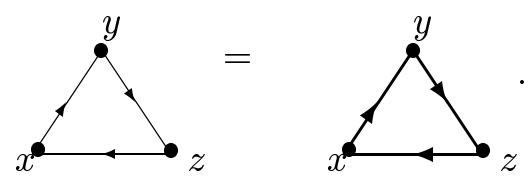

Moreover, the barycentric property of $g$ reads
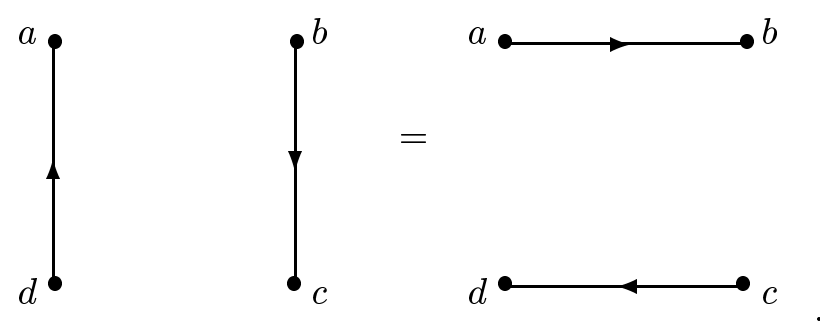

Hence
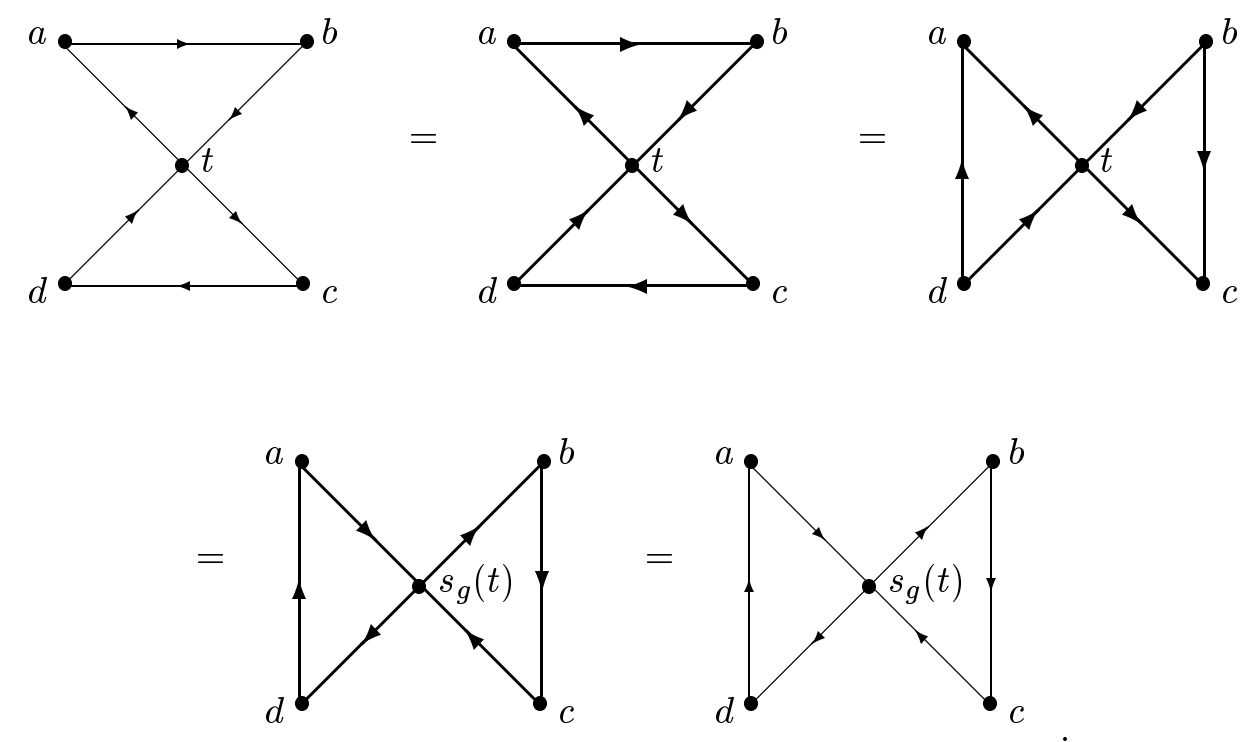
One can therefore choose our diffeomorphism $\varphi$ as

$$
\varphi=s_{g}
$$

\section{Remarks 1.4.}

(i) Given four points $\{a, b, c, d\}$ in the Euclidean plane $E^{2}$, one can define two smooth functions $F$ and $G \in C^{\infty}\left(E^{2}, \mathbb{R}\right)$ as follows. For $t \in E^{2}$, $G(t)$ is the sum of the signed Euclidean areas of the oriented Euclidean triangles $\Delta a b t$ and $\Delta d t c$, i.e.,

$$
G(t)=S A(\Delta a b t)+S A(\Delta d t c)
$$

where, for a closed path $\gamma$ in $E^{2}$, one sets $S A(\gamma)=\int_{\gamma} x d y$.

Similarly, one defines for the other pair of triangles $\Delta d a t$ and $\Delta b c t$,

$$
F(t)=S A(\Delta d a t)+S A(\Delta b c t)
$$

or equivalently, if $S A(a b c d)$ denotes the signed area of the oriented quadrilateron with vertices $a, b, c, d$,

$$
F(t)=S A(a b c d)-G(t) .
$$

If our four points are the vertices of a square in $E^{2}$, one obviously has the equality

$$
F=G \text {. }
$$

For arbitrary quadrilaterons, equality between $F$ and $G$ does not generally hold. But the proof of Proposition 1.3 leads to a natural generalization:

For every quadruple of points $\{a, b, c, d\}$ in $E^{2}$, one has

$$
F=s_{g}^{\star} G
$$

where $g$ is the center of mass of the quadrilateron abcd, and, where $s_{g}: E^{2} \rightarrow E^{2}$ is the Euclidean symmetry $s_{g}(x)=2 g-x$.

Proposition 1.3 tells us that this elementary property of Euclidean quadrilaterons of $E^{2}$ is the geometric content of associativity of Weyl's product. Indeed, associativity for the Weyl product (on $\mathbb{R}^{2}$ ) reads (cf. formula (4)):

$$
\int_{\mathbb{R}^{2}} \exp i F(t) d t=\int_{\mathbb{R}^{2}} \exp i G(\tau) d \tau .
$$

This equality being realized by the change of variables $\tau=s_{g}(t)$. 
(ii) Proposition 1.3 also leads to a geometric understanding of the associativity of Rieffel's deformed product obtained from an action of $\mathbb{R}^{d}$ on a $C^{*}$-algebra [21]. This is based on the following observation.

Let $G$ be a Lie group, endowed with a left invariant Haar measure $\mu$. Assume that there exists a geometrically associative three-point kernel $K \in C^{\infty}(G \times G \times G)$ (cf. Definition 1.1) on $(G, \mu)$ which is invariant under the diagonal action of $G$ on $G \times G \times G$ by left translations. Then, the data of an action $\alpha: G \times A \rightarrow A$ of $G$ on some (topological) associative algebra $A$ by automorphisms yields a new associative product on $A$, provided the situation is sufficiently regular. Indeed, on just observes that the geometric associativity of $K$ implies, at least formally, associativity of the product $\star$ on $A$ defined by the formula:

$$
a \star b=\int_{G \times G} K(e, g, h) \alpha_{g}(a) \alpha_{h}(b),
$$

where $a, b \in A$, and where integration is taken over $g$ and $h$ with respect to the measure $\mu \otimes \mu$ on $G \times G$. In the case of an action of the Abelian group $G=\mathbb{R}^{d}$, endowed with the Euclidean scalar product $\langle$,$\rangle , every skewsymmetric matrix J \in s o\left(\mathbb{R}^{d}\right)$ yields an invariant threepoint function $S^{J} \in C^{\infty}\left(\mathbb{R}^{d} \times \mathbb{R}^{d} \times \mathbb{R}^{d}\right)$ via the formula

$$
S^{J}(x, y, z)=\langle x, J y\rangle+\langle y, J z\rangle+\langle z, J x\rangle .
$$

Defining the Euclidean symmetries of $\mathbb{R}^{d}$ as $s_{x}(y)=2 x-y$, one gets a Weyl triple $\left(\mathbb{R}^{d}\right.$, Haar, $\left.S^{J}\right)$ (Definition 1.2). Hence, by Proposition 1.3, an invariant geometrically associative kernel

$$
K=e^{i S^{J}}
$$

When given an action $\alpha: \mathbb{R}^{d} \times A \rightarrow A$, formula (5) with $K=e^{i S^{J}}$ yields Rieffel's product in [21].

(iii) In the case of the hyperbolic plane endowed with its natural structure of symmetric space, it is tempting to ask whether the three-point function defined by the symplectic area $S A(x, y, z)$ of the geodesic triangle $\Delta x y z$ would satisfy properties (i) - (iii) in Definition 1.2. The answer is negative. Indeed, properties (ii) and (iii) would imply unboundedness of $S A$. This indicates that, when requiring some compatibility between $S$ and the symmetries, property (iii) is somehow too strong. 
Proposition 1.3 and Remark 1.4 lead us to consider a class of manifolds which carry a large family of "symmetries". Symplectic symmetric spaces are defined in Section 2. They constitute a class of affine symplectic manifolds. We will now make precise what we mean by "WKB-quantization" in the context of affine symplectic manifolds.

Let $(M, \omega, \nabla)$ be a $2 n$-dimensional affine symplectic manifold, that is, $(M, \omega)$ is a smooth connected symplectic manifold and $\nabla$ is an torsionfree affine connection on $M$ such that $\nabla \omega=0$. Its automorphism group $\operatorname{Aut}(M, \omega, \nabla)$ is defined as

$$
\operatorname{Aut}(M, \omega, \nabla)=\operatorname{Aff}(\nabla) \cap \operatorname{Symp}(\omega)
$$

where $\operatorname{Aff}(\nabla)$ is the group of affine transformations of the affine manifold $(M, \nabla)$ and where $\operatorname{Symp}(\omega)$ denotes the group of symplectomorphisms of $(M, \omega)$. Note that, since $\operatorname{Aff}(\nabla)$ is a Lie group of transformations of $M$ (cf. [13]), so is $\operatorname{Aut}(M, \omega, \nabla)$.

Definition 1.5. Let $G$ be a subgroup of $\operatorname{Aut}(M, \omega, \nabla)$. A $G$-invariant WKB-quantization of $(M, \omega, \nabla)$ is a triple $\left(\left\{\left(\mathcal{E}_{\hbar}, \star_{\hbar}\right)\right\}_{\hbar \geq 0}, S,\left\{a_{\hbar}\right\}_{\hbar \geq 0}\right)$ satisfying the following properties.

(i) $\left\{\left(\mathcal{E}_{\hbar}, \star_{\hbar}\right)\right\}_{\hbar \geq 0}$ is a one-parameter family of associative ${ }^{*}$-algebras such that:

(i.1) $\left(\mathcal{E}_{0}, \star_{0}\right)$ is a Poisson subalgebra of $C^{\infty}(M)$ endowed with the usual pointwize multiplication of functions (the Poisson structure $\{$, is the one associated to the symplectic form $\omega$ ).

(i.2) For all $\hbar \geq 0, \mathcal{E}_{\hbar}$ is a ${ }^{*}$-linear subspace of $C^{\infty}(M)$ such that the following inclusions hold

$$
\mathcal{D}(M) \subset \mathcal{E}_{0} \subset \mathcal{E}_{\hbar}
$$

where $\mathcal{D}(M)$ denotes the space of smooth compactly supported functions on $M$ and where on $C^{\infty}(M)$ the involution is the complex conjugation.

(ii) $S$ is a real valued smooth three-point function: $S \in C^{\infty}(M \times M \times M, \mathbb{R})$ such that, for all $x_{0} \in M$ the partial function $S\left(x_{0}, .,.\right) \in C^{\infty}(M \times$ $M, \mathbb{R})$ has an nondegenerate critical point at $\left(x_{0}, x_{0}\right) \in M \times M$. One furthermore requires the function $S$ to be invariant under the diagonal action of $G$ on $M \times M \times M$. 
(iii) $\left\{a_{\hbar}\right\}_{\hbar \geq 0}$ is a smooth (with respect to $\hbar$ ) family of positively real valued three-point functions: $a_{\hbar} \in C^{\infty}\left(M \times M \times M, \mathbb{R}^{+}\right)$which are invariant under the diagonal action of $G$ on $M \times M \times M$.

(iv) At the level of the subspace $\mathcal{D}(M) \subset \mathcal{E}_{\hbar}(\hbar>0)$, the multiplication $\star \hbar$ reads

$$
\left(u \star_{\hbar} v\right)(x)=\frac{1}{\hbar^{2 n}} \int_{M \times M} a_{\hbar}(x, y, z) \exp \left(\frac{i}{\hbar} S(x, y, z)\right) u(y) v(z) d y d z
$$

where $u, v \in \mathcal{D}(M)$ and where $d y$ (and $d z$ ) stands for the Liouville measure $\frac{\omega^{n}}{n !}$.

(v) For all $x \in M$ and all $u, v \in \mathcal{D}(M) \subset \mathcal{E}_{\hbar}$ supported in a sufficiently small neighborhood of $x$, a stationary phase method yields the following asymptotic expansion (cf. [15]):

$$
(u \star \hbar v)(x) \sim u(x) v(x)+\frac{\hbar}{i} c_{1}(u, v)(x)+o\left(\hbar^{2}\right)
$$

with

$$
\frac{1}{2}\left(c_{1}(u, v)(x)-c_{1}(v, u)(x)\right)=\{u, v\}(x)
$$

This definition is very much inspired from Rieffel's definition of strict quantization. However, the topological framework is weaker than Rieffel's one. For instance, the deformed algebras are function algebras but they do not a priori carry any topological structure. Also Dirac's condition does only hold at the formal level (see item $(v)$ in Definition 1.5). Note moreover that each function space $\mathcal{E}_{\hbar}$ is not required to be invariant under the action of $G$ on $C^{\infty}(M)$. However, the space of compactly supported functions $\mathcal{D}(M)$ is $G$-invariant, and, by invariance of the functions $a_{\hbar}$ and $S$, one will always have

$$
g\left(u \star_{\hbar} v\right)=(g u) \star_{\hbar}(g v) \quad \forall g \in G
$$

as soon as $u, v \in \mathcal{D}(M) \subset \mathcal{E}_{\hbar}$.

\section{General facts about symplectic symmetric spaces.}

Definition 2.1 $([5,6])$. A symplectic symmetric space is a triple $(M, \omega, s)$, where $(M, \omega)$ is a smooth connected symplectic manifold, and where $s: M \times M \rightarrow M$ is a smooth map such that 
(i) for all $x$ in $M$, the partial map $s_{x}: M \rightarrow M: y \mapsto s_{x}(y):=s(x, y)$ is an involutive symplectic diffeomorphism of $(M, \omega)$ called the symmetry at $x$.

(ii) For all $x$ in $M, x$ is an isolated fixed point of $s_{x}$.

(iii) For all $x$ and $y$ in $M$, one has $s_{x} s_{y} s_{x}=s_{s_{x}(y)}$.

Definition 2.2. Two symplectic symmetric spaces $(M, \omega, s)$ and $\left(M^{\prime}, \omega^{\prime}, s^{\prime}\right)$ are isomorphic if there exists a symplectic diffeomorphism $\varphi:(M, \omega) \rightarrow$ $\left(M^{\prime}, \omega^{\prime}\right)$ such that $\varphi s_{x}=s_{\varphi(x)}^{\prime} \varphi$. Such a $\varphi$ is called an isomorphism of $(M, \omega, s)$ onto $\left(M^{\prime}, \omega^{\prime}, s^{\prime}\right)$. When $(M, \omega, s)=\left(M^{\prime}, \omega^{\prime}, s^{\prime}\right)$, one talks about automorphisms. The group of all automorphisms of the symplectic symmetric space $(M, \omega, s)$ is denoted by $\operatorname{Aut}(M, \omega, s)$.

Proposition 2.3. On a symplectic symmetric space $(M, \omega, s)$, there exists one and only one affine connection $\nabla$ which is invariant under the symmetries. Moreover, this connection satisfies the following properties.

(i) For all smooth tangent vector fields $X, Y, Z$ on $M$ and all points $x$ in $M$, one has

$$
\omega_{x}\left(\nabla_{X} Y, Z\right)=\frac{1}{2} X_{x} \cdot \omega\left(Y+s_{x_{\star}} Y, Z\right) .
$$

(ii) $(M, \nabla)$ is an affine symmetric space. In particular $\nabla$ is torsion free and its curvature tensor is parallel.

(iii) The symplectic form $\omega$ is parallel; $\nabla$ is therefore a symplectic connection.

(iv) One has

$$
\operatorname{Aut}(M, \omega, s)=\operatorname{Aut}(M, \omega, \nabla)=\operatorname{Aff}(\nabla) \cap \operatorname{Symp}(\omega) .
$$

The connection $\nabla$ on the symmetric space $(M, s)$ is called the Loos connection. The following facts are classical (see [14], v. I, [13], v. II, Chapters X and XI).

Theorem 2.4. Let $(M, \omega, s)$ be a symplectic symmetric space and $\nabla$ its Loos connection. Fix $o$ in $M$ and denote by $H$ the stabilizer of $o$ in Aut $(M, \omega, s)$. Denote by $G$ the transvection group of $(M, s)$ (i.e., the subgroup of $\operatorname{Aut}(M, \omega, s)$ generated by $\left.\left\{s_{x} \circ s_{y} ; x, y \in M\right\}\right)$ and set $K=G \cap H$. Then, 
(i) the transvection group $G$ turns out to be a connected Lie transformation group of $M$. It is the smallest subgroup of Aut $(M, \omega, s)$ which is transitive on $M$ and stabilized by the conjugation $\tilde{\sigma}: \operatorname{Aut}(M, \omega, s) \rightarrow$ Aut $(M, \omega, s)$ defined by $\tilde{\sigma}(g)=s_{o} g s_{o}$.

(ii) The homogeneous space $M=G / K$ is reductive. The Loos connection $\nabla$ coincide with the canonical connection induced by the structure of reductive homogeneous space.

(iii) Denoting by $G^{\tilde{\sigma}}$ the set of $\tilde{\sigma}$-fixed points in $G$ and by $G_{0}^{\tilde{\sigma}}$ its neutral connected component, one has

$$
G_{0}^{\tilde{\sigma}} \subset K \subset G^{\tilde{\sigma}} .
$$

Moreover, the Lie algebra $\mathcal{K}$ of $K$ is isomorphic to the holonomy algebra with repect to the canonical connection $\nabla$.

(iv) Denote by $\sigma$ the involutive automorphism of the Lie algebra $\mathcal{G}$ of $G$ induced by the automorphism $\tilde{\sigma}$. Denote by $\mathcal{G}=\mathcal{K} \oplus \mathcal{P}$ the decomposition in \pm 1 -eigenspaces for $\sigma$. Then, identifying $\mathcal{P}$ with $T_{o}(M)$, one has

$$
\exp (X)=s_{\operatorname{Exp}_{o}\left(\frac{1}{2} X\right)} \circ s_{o}
$$

for all $X$ in a neighborhood of 0 in $\mathcal{P}$. Here exp is the exponential map $\exp : \mathcal{G} \rightarrow G$ and $\operatorname{Exp}_{o}$ is the exponential map at point $o$ with respect to the connection $\nabla$.

Definition 2.5. Let $(\mathcal{G}, \sigma)$ be an involutive algebra, that is, $\mathcal{G}$ is a finite dimensional real Lie algebra and $\sigma$ is an involutive automorphism of $\mathcal{G}$. Let $\Omega$ be a skewsymmetric bilinear form on $\mathcal{G}$. Then the triple $(\mathcal{G}, \sigma, \Omega)$ is called a symplectic triple if the following properties are satisfied.

(i) Let $\mathcal{G}=\mathcal{K} \oplus \mathcal{P}$ where $\mathcal{K}$ (resp. $\mathcal{P}$ ) is the +1 (resp. -1) eigenspace of $\sigma$. Then $[\mathcal{P}, \mathcal{P}]=\mathcal{K}$ and the representation of $\mathcal{K}$ on $\mathcal{P}$, given by the adjoint action, is faithful.

(ii) $\Omega$ is a Chevalley 2-cocycle for the trivial representation of $\mathcal{G}$ on $\mathbb{R}$ such that for any $X$ in $\mathcal{K}, i(X) \Omega=0$. Moreover, the restriction of $\Omega$ to $\mathcal{P} \times \mathcal{P}$ is nondegenerate.

The dimension of $\mathcal{P}$ defines the dimension of the triple. Two such triples $\left(\mathcal{G}_{i}, \sigma_{i}, \Omega_{i}\right)(i=1,2)$ are isomorphic if there exists a Lie algebra isomorphism $\psi: \mathcal{G}_{1} \rightarrow \mathcal{G}_{2}$ such that $\psi \circ \sigma_{1}=\sigma_{2} \circ \psi$ and $\psi^{*} \Omega_{2}=\Omega_{1}$. 
Theorem 2.4 associates to a symplectic symmetric space $(M, \omega, s)$ an involutive Lie algebra $(\mathcal{G}, \sigma)$. Denoting by $\pi: G \rightarrow M$ the natural projection, one checks that the triple $\left(\mathcal{G}, \sigma, \Omega=\pi^{*}\left(\omega_{o}\right)\right)$ is a symplectic triple. This implies the next proposition.

Proposition 2.6. There is a bijective correspondence between the isomorphism classes of simply connected symplectic symmetric spaces $(M, \omega, s)$ and the isomorphism classes of symmetric triples $(\mathcal{G}, \sigma, \Omega)$.

Since a symmetric symplectic manifold $(M, \omega, s)$ is a symplectic homogeneous space of its transvection group $G$, it seems natural, when possible, to relate $(M, \omega, s)$ to a coadjoint orbit of $G$ in $\mathcal{G}^{\star}$. Recall first the two following definitions.

Definition 2.7. Let $G$ be a Lie group of symplectomorphisms acting on a symplectic manifold $(M, \omega)$. For every element $X$ in the Lie algebra $\mathcal{G}$ of $G$, one denotes by $X^{*}$ the fundamental vector field associated to $X$, i.e., for $x$ in $M$,

$$
X_{x}^{*}=\left.\frac{d}{d t} \exp (-t X) x\right|_{t=0} .
$$

The action is called weakly Hamiltonian if for all $X$ in $\mathcal{G}$ there exists a smooth function $\lambda_{X} \in C^{\infty}(M)$ such that

$$
i\left(X^{*}\right) \omega=d \lambda_{X}
$$

In this case, if the correspondence $\mathcal{G} \rightarrow C^{\infty}(M): X \mapsto \lambda_{X}$ is also a homomorphism of Lie algebras, one says that the action of $G$ on $(M, \omega)$ is Hamiltonian. (The Lie algebra structure on $C^{\infty}(M)$ is defined by the Poisson bracket.)

Proposition 2.8. Let $t=(\mathcal{G}, \sigma, \Omega)$ be a symplectic triple and let $(M, \omega, s)$ be the associated simply connected symplectic symmetric space. The action of the transvection group $G$ on $M$ is Hamiltonian if and only if $\Omega$ is a Chevalley coboundary, that is, there exists an element $\xi$ in $\mathcal{G}^{*}$ such that $\Omega=\delta \xi$. In this case, $(M, \omega, s)$ is a $G$-equivariant symplectic covering of $\mathcal{O}$, the coadjoint orbit of $\xi$ in $\mathcal{G}^{\star}$.

The action of the transvection group $G$ is in general not Hamiltonian. We therefore need to consider a one-dimensional central extension of $G$ rather than $G$ itself. At the infinitesimal level, this corresponds to extending the algebra $\mathcal{G}$ by the 2 -cocycle $\Omega$. This way, one associates to any symplectic symmetric space an exact triple in the following sense (see [7] for details). 
Definition 2.9. An exact triple is a triple $\tau=(\mathcal{G}, \sigma, \Omega)$, where

(i) $(\mathcal{G}, \sigma)$ is an involutive Lie algebra such that, if $\mathcal{G}=\mathcal{K} \oplus \mathcal{P}$ is the decomposition with respect to $\sigma$, one has $[\mathcal{P}, \mathcal{P}]=\mathcal{K}$.

(ii) $\Omega$ is a Chevalley 2-coboundary (i.e., $\Omega=\delta \xi, \quad \xi \in \mathcal{G}^{\star}$ ) such that $i(\mathcal{K}) \Omega=0$ and $\left.\Omega\right|_{\mathcal{P} \times \mathcal{P}}$ is symplectic.

Remark 2.10. (i) One can choose $\xi \in \mathcal{G}^{\star}$ such that $\xi(\mathcal{P})=0$. One therefore writes, with a slight abuse of notation, $\xi \in \mathcal{K}^{\star}$.

(ii) Observe that, when associated to a (transvection) symplectic triple, the center $\mathcal{Z}(\mathcal{G})$ of the Lie algebra $\mathcal{G}$ occurring in an exact triple is at most one dimensional. Indeed, on the one hand, exactness implies $\mathcal{Z}(\mathcal{G}) \subset \mathcal{K}$. One the other hand, faithfulness of the holonomy representation forces $\operatorname{dim}(\mathcal{Z}(\mathcal{G}) \cap \mathcal{K}) \leq 1$ since $\mathcal{K}$ is either the holonomy algebra itself or a one dimensional central extension.

\section{Phase functions on symplectic symmetric spaces.}

Motivated by the definition of a Weyl triple (Definition 1.2), as well as by the third part of Remarks 1.4, we now make the following definition, adapting to symmetric spaces the notion of phase function.

Definition 3.1. Let $(M, \omega, s)$ be a symplectic symmetric space (see Definition 2.1). A smooth function $S: M \times M \times M \rightarrow \mathbb{R}$ satisfying the following properties:

(i) $S(x, y, z)=S(z, x, y)=-S(y, x, z)$;

(ii) the function $S$ is invariant under the symmetries i.e.,

$$
S\left(s_{m}(x), s_{m}(y), s_{m}(z)\right)=S(x, y, z) \quad \forall x, y, z, m \in M
$$

(iii) for all $x \in M$, the symmetry $s_{x}: M \rightarrow M$ is such that

$$
S(x, y, z)=-S\left(x, s_{x}(y), z\right) \quad \forall y, z \in M
$$

is called an admissible function. 
In [25], Weinstein proved that, in the case of a Hermitian symmetric space $(M, \omega, s)$ of the noncompact type, the phase function $S_{W}$, occurring in the expression of a given invariant WKB-quantization of $(M, \omega)$ defined by an oscillatory integral formula of the type

$$
u \star v(x)=\int u(y) v(z) a_{\hbar}(x, y, z) e^{\frac{i}{\hbar} S_{W}(x, y, z)} d y d z,
$$

must be as follows.

- Let $x, y$ and $z$ be three points in $M$ such that the following equation admits a solution $t$ :

$$
t=s_{x} s_{y} s_{z}(t)
$$

( $t$ is unique if it exists).

- Let $\Sigma$ be a surface in $M$ bounded by the geodesic triangle $\Delta t A B$ where $A=s_{x}(t)$ and $B=s_{y}(A)=s_{y} s_{x}(t) \quad\left(t=s_{z}(B)\right)$.

Then, if for some formal amplitude of the form

$$
a_{\hbar}(x, y, z)=a_{0}(x, y, z)+\hbar a_{1}(x, y, z)+\hbar^{2} a_{2}(x, y, z)+\ldots,
$$

the product (7) defines an invariant deformation quantization of $(M, \omega)$, the value of the "WKB"-phase function $S_{W}$ on $(x, y, z)$ is given by

$$
S_{W}(x, y, z)=-\int_{\Sigma} \omega
$$

Practically, the function $S_{W}$ is hard to compute explicitly; however, some two dimensional examples have been treated in [18]. The problem of finding the amplitude $a_{\hbar}$ is open.

Proposition 3.2. The function $S_{W}$ is admissible.

Proof. Let $x, y$ and $z$ be three points in $M$, and set

$$
\left\{\begin{aligned}
Y & =s_{z} s_{y} s_{x}(Y) \\
Z & =s_{x}(Y) \\
X & =s_{y} s_{x}(Y)=s_{y}(Z)
\end{aligned}\right.
$$

and

$$
\left\{\begin{array}{l}
\zeta=s_{s_{x}(y)} s_{z} s_{x}(\zeta) \quad(* *) \\
\xi=s_{x}(\zeta) \\
\eta=s_{z} s_{x}(\zeta)=s_{z}(\xi)
\end{array}\right.
$$


(provided equations $(*)$ and $\left({ }^{*}\right)$ admit solutions). The only thing we really need to show is that the function $S_{W}$ satisfies property (iii) in Definition 3.1; that is,

$$
S A(X, Y, Z)=S A(\xi, \eta, \zeta),
$$

where the function $S A$ is defined as follows. For a sequence of points $\left\{x_{i}\right\}_{0 \leq i \leq N}$, the expression $S A\left(x_{0}, \ldots, x_{N}\right)$ means $S A(\gamma)=\int_{\gamma} \alpha$, where $\gamma$ is the piecewise geodesic path whose $i^{\text {th }}$ geodesic segment starts from point $x_{i}$ and ends at point $x_{i+1 \bmod (N+1)}$, and where $\alpha$ is a 1-form such that $\omega=d \alpha$.

On the first hand, one has: $s_{x} s_{s_{x}(y)} s_{z}(X)=s_{x} s_{x} s_{y} s_{x} s_{z}(X)=$ $s_{y} s_{x} s_{z}(X)=X$. Hence $X=\xi$ and $s_{s_{x}(z)}(Z)=\zeta$. On the second hand, the invariance of $S A$ under the symmetries yields: $S A(\xi, \eta, \zeta)=$ $S A\left(s_{x}(\xi), s_{x}(\eta), s_{x}(\zeta)\right)=S A(\zeta, Z, \xi)$. Moreover, $S A(\xi, \eta, \zeta)+S A(\zeta, Z, \xi)=$ $S A(\xi, \eta, \zeta, Z)$, hence

$$
S_{W}\left(x, s_{x}(y), z\right)=\frac{1}{2} S A(\xi, \eta, \zeta, Z) .
$$

Similarly, one gets

$$
S_{W}(x, y, z)=-\frac{1}{2} S A(\xi, \eta, \zeta, Z) .
$$

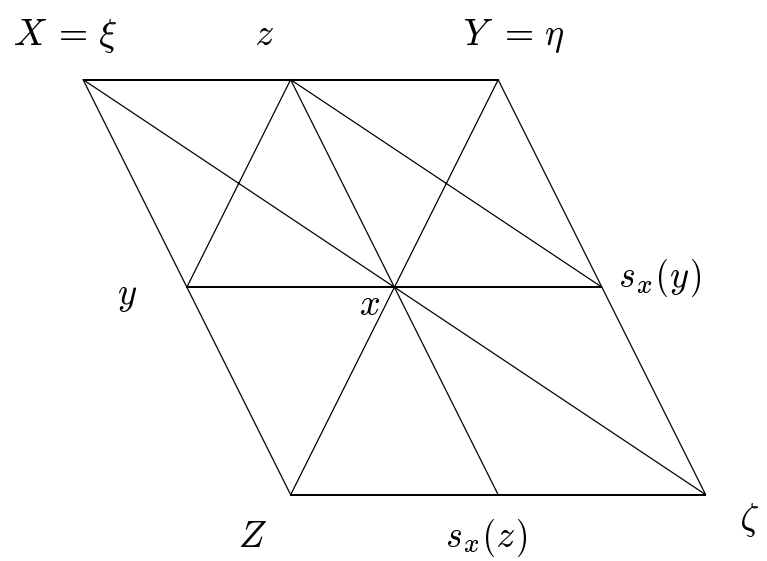


Now, suppose we are given, on a symplectic symmetric space $(M, \omega, s)$, an admissible three-point function $S$. The choice of a base point $o$ in $M$ then determines a two-point function $u$ on $M \times M$ :

$$
u(x, y)=S(o, x, y)
$$

The following proposition tells us, in the case where $(M, \nabla)$ is strictly geodesically convex, when a given two-point function comes from an admissible three-point function.

Proposition 3.3. Let $(M, \omega, s)$ be a symplectic symmetric space and let $o$ be a point in $M$. Assume the existence of a smooth midpoint map, that is, a map $M \rightarrow M: x \rightarrow \frac{x}{2}$ such that $s_{\frac{x}{2}}(o)=x$. Denote by Stab(o) the stabilizer of $o$ in the automorphism group of $(M, \omega, s)$. Let $u: M \times M \rightarrow \mathbb{R}$ be a Stab(o)-invariant smooth two-point function such that

$$
u(x, y)=-u(y, x)=-u\left(x, s_{x}(y)\right)=-u\left(x, s_{\frac{x}{2}}(y)\right) \quad \forall x, y \in M .
$$

Then, the three-point function $S$ defined by

$$
S(x, y, z)=u\left(s_{\frac{x}{2}}(y), s_{\frac{x}{2}}(z)\right)
$$

is admissible.

Proof. The function $S$ is invariant under the symmetries. Indeed, let $g$ be an element of the group of transformations of $M$ generated by the symmetries. For all $x$ in $M$, one has $s_{\frac{g x}{2}}(o)=g x$ that is $g^{-1} s_{\frac{g x}{2}}(o)=s_{\frac{x}{2}}(o)$; hence $s \frac{x}{2} g^{-1} s \frac{g x}{2}$ is an element of Stab $(o)$. By invariance under the action of the stabilizer, one has

$$
\begin{aligned}
& u\left(s_{\frac{g x}{2}}(g y), s_{\frac{g x}{2}}(g z)\right) \\
& =u\left(s_{\frac{x}{2}} g^{-1} s_{\frac{g x}{2}} s_{\frac{g x}{2}}(g y), s \frac{x}{2} g^{-1} s \frac{g x}{2} s_{\frac{g x}{2}}(g z)\right) \\
& =u\left(s_{\frac{x}{2}}(y), s_{\frac{x}{2}}(z)\right) .
\end{aligned}
$$




$$
\begin{aligned}
& \text { Now, since } s_{\frac{x}{2}} s_{\frac{z}{2}} s\left(\frac{s_{\frac{z}{2}}(x)}{2}\right) \text { is an element of } \operatorname{Stab}(o) \text {, one has } \\
& u\left(s_{\frac{z}{2}}(x), s_{\frac{z}{2}}(y)\right)=-u\left(s_{\frac{z}{2}}(x), s\left(\frac{s_{\frac{z}{2}}(x)}{2}\right) s_{\frac{z}{2}}(y)\right) \\
& =-u\left(s_{\frac{x}{2}} s \frac{z}{2} s\left(\frac{s_{\frac{z}{2}}(x)}{2}\right) s \frac{z}{2}(x), s \frac{x}{2} s \frac{z}{2} s\left(\frac{s_{\frac{z}{2}}(x)}{2}\right) s^{s}\left(\frac{s_{\frac{z}{2}}(x)}{2}\right) s^{\frac{z}{2}}(y)\right) \\
& =-u\left(s_{\frac{x}{2}} s_{\frac{z}{2}} s\left(\frac{s_{\frac{z}{2}}(x)}{2}\right) s_{\frac{z}{2}} S \frac{x}{2}(o), s \frac{x}{2}(y)\right) \\
& =-u\left(s_{\frac{x}{2}} s_{\frac{z}{2}}\left(s_{\frac{x}{2}} s_{\frac{z}{2}} s\left(\frac{s_{\frac{z}{2}}(x)}{2}\right)\right)^{-1}(o), s_{\frac{x}{2}}(y)\right) \\
& =-u\left(s_{\frac{x}{2}}(z), s_{\frac{x}{2}}(y)\right)=u\left(s_{\frac{x}{2}}(y), s_{\frac{x}{2}}(z)\right) .
\end{aligned}
$$

This shows that the function $S$ satisfies the condition $(i)$ in Definition 3.1. The remaining condition is obviously satisfied.

Definition 3.4. Let $(M, \omega, s)$ be a symplectic symmetric space admitting a midpoint map (cf. Proposition 3.3) with respect to a point $o$ in $M$. An $o$-admissible two-point function on $M$ is a $S t a b(o)$-invariant smooth function $u: M \times M \rightarrow \mathbb{R}$ such that

$$
u(x, y)=-u(y, x)=-u\left(x, s_{x}(y)\right)=-u\left(x, s_{\frac{x}{2}}(y)\right) \quad \forall x, y \in M .
$$

It turns out that admissible two-point functions are easy to determine.

Example 3.5. The triple $(M, \omega, s)=\left(\mathbb{R}^{2}, d p \wedge d q, s\right)$ with

$$
s_{(p, q)}\left(p^{\prime}, q^{\prime}\right)=\left(2 p-p^{\prime}, 2 \cosh \left(p-p^{\prime}\right) q-q^{\prime}\right)
$$

defines a (non-metric) symplectic symmetric space [7], [5]. It is strictly geodesically convex. One checks that the function $u: M \times M \rightarrow \mathbb{R}$ defined by

$$
u\left((p, q),\left(p^{\prime}, q^{\prime}\right)\right)=\sinh \left(p^{\prime}\right) q-\sinh (p) q^{\prime}
$$

is an $(0,0)$-admissible two-point function whose corresponding admissible three-point function $S$ is given by:

$$
\begin{aligned}
& S\left((p, q),\left(p^{\prime}, q^{\prime}\right),\left(p^{\prime \prime}, q^{\prime \prime}\right)\right) \\
& =\sinh \left(p-p^{\prime}\right) q^{\prime \prime}+\sinh \left(p^{\prime \prime}-p\right) q^{\prime}+\sinh \left(p^{\prime}-p^{\prime \prime}\right) q .
\end{aligned}
$$




\section{Elementary solvable symplectic symmetric spaces.}

In Definition 4.1 below, we define a particular type of solvable symmetric spaces that we call elementary. It has been proven ([7], Proposition 3.2) that every solvable symmetric space can be expressed as the result of a sequence of split extensions by Abelian (flat) factors successively taken over an elementary solvable symmetric space. We therefore consider elementary solvable symmetric spaces as the "first induction step" when studying solvable symmetric spaces.

Definition 4.1. A symplectic symmetric space $(M, \omega, s)$ is called an elementary solvable symplectic symmetric space if its associated exact triple $(\mathcal{G}, \sigma, \Omega=\delta \xi$ ) (see Definition 2.9) is of the following type.

(i) The Lie algebra $\mathcal{G}$ is a split extension of Abelian Lie algebras $\mathcal{A}$ and $\mathcal{B}$ :

$$
\mathcal{B} \longrightarrow \mathcal{G} \leftrightarrows \mathcal{A}
$$

(ii) The automorphism $\sigma$ preserves the splitting $\mathcal{G}=\mathcal{B} \oplus \mathcal{A}$.

Such an exact triple (associated to an elementary solvable symplectic symmetric space) is called an elementary solvable exact triple (briefly: ESET).

Observe that, since $\mathcal{A} \cap \mathcal{K} \subset \mathcal{A} \cap[\mathcal{G}, \mathcal{G}]=0$, one has $\mathcal{A} \subset \mathcal{P}$. Therefore $\mathcal{B}=\mathcal{K} \oplus \mathcal{L}$, with $\mathcal{L} \subset \mathcal{P}$. Moreover, since $\mathcal{L}$ and $\mathcal{A}$ are Abelian and $\Omega$ is nondegenerate, the subspaces $\mathcal{A}$ and $\mathcal{L}$ of $\mathcal{P}$ are Lagrangians in duality.

Now, let $\rho: \mathcal{A} \rightarrow \operatorname{End}(\mathcal{B})$ be the splitting homomorphism. The group law on $G$ identified with $\mathcal{G}=\mathcal{A} \times \mathcal{B}$ is given by

$$
(a, b) \cdot\left(a^{\prime}, b^{\prime}\right)=\left(a+a^{\prime}, \exp (\rho(a)) b^{\prime}+b\right) .
$$

The (simply connected) symplectic symmetric space $(M, \omega, s)$ associated to an $\operatorname{ESET}(\mathcal{G}, \sigma, \Omega)$ is described as follows $[5,7]$.

\section{Proposition 4.2.}

(i) The homogeneous space $G \rightarrow M=G / K$ is realized by the diagram

$$
G=\mathcal{A} \times \mathcal{K} \times \mathcal{L} \stackrel{\pi}{\longrightarrow} \mathcal{P}=\mathcal{A} \times \mathcal{L}
$$

where

$$
\pi(a, k, l)=(a,-\sinh (a) k+\cosh (a) l)
$$


with $\sinh (a) \stackrel{\text { def. }}{=} \frac{1}{2}(\exp \rho(a)-\exp \rho(-a))$ and $\cosh (a) \stackrel{\text { def. }}{=} \frac{1}{2}(\exp \rho(a)+$ $\exp \rho(-a))$. The map $\gamma: \mathcal{P} \rightarrow G$ given by

$$
\gamma(a, l)=(a, \sinh (a) l, \cosh (a) l)
$$

defines a global section of the principal bundle $(M, G, \pi)$. The action of $G$ on $M=\mathcal{P}$ reads as follows:

$$
(\alpha, \kappa, \lambda)(a, l)=(a+\alpha, \cosh (a+\alpha) \lambda-\sinh (a+\alpha) \kappa+l) .
$$

(ii) The identification $M=\mathcal{P}$ defines a global Darboux $\operatorname{chart}(M, \omega) \stackrel{\sim}{\rightarrow}$ $(\mathcal{P}, \Omega)$. In this chart, the symmetries are given by:

$$
s_{(a, l)}\left(a^{\prime}, l^{\prime}\right)=\left(2 a-a^{\prime}, 2 \cosh \left(a-a^{\prime}\right) l-l^{\prime}\right) .
$$

If $o=\pi(0,0,0)=(0,0)$, the midpoint map $M \rightarrow M: x \rightarrow \frac{x}{2} \quad\left(s_{\frac{x}{2}} O=\right.$ $x)$ is given (locally) by:

$$
\frac{(a, l)}{2}=\frac{1}{2}\left(a,\left(\cosh \frac{1}{2} a\right)^{-1} l\right) .
$$

The following map will be important while defining the deformed product.

Definition 4.3. Let $(\mathcal{G}, \sigma, \Omega=\delta \xi)$ be an ESET (cf. Definition 4.1). We denote by $\zeta$ the "pairing":

$$
\zeta: \mathcal{A} \times \mathcal{L} \rightarrow \mathbb{R}
$$

defined by

$$
\zeta(a, l)=\xi(\sinh (a) l)
$$

The formula

$$
\omega(\phi(a), l)=\zeta(a, l)
$$

defines a "linearization" map $\phi: \mathcal{A} \rightarrow \mathcal{A}$, called the twisting map.

Proposition 4.4. Let $(M, \omega, s)$ be an elementary solvable symplectic symmetric space. Set $\omega=\Omega=\delta \xi\left(\xi \in \mathcal{K}^{*}\right.$ ) (see Proposition 4.2 (ii)). Let $\mathcal{A} \times \mathcal{L} \stackrel{\omega}{\rightarrow} \mathbb{R}$ be the symplectic pairing:

$$
\omega(a, l)=\Omega(a, l)=-\xi[a, l] .
$$

Then, the midpoint map $M \rightarrow M: x \rightarrow \frac{x}{2} \quad\left(s_{\frac{x}{2}} O=x\right)$ is globally defined provided the twisting map $\phi: \mathcal{A} \rightarrow \mathcal{A}$ is a diffeomorphism. 
Proof. One has a linear isomorphism $\mathcal{A} \stackrel{\Omega}{\rightarrow} \mathcal{L}^{\star}$ defined by $\langle\Omega(a), l\rangle=$ $\omega(a, l)$. Also, since $\zeta: \mathcal{A} \times \mathcal{L} \rightarrow \mathbb{R}$ is linear in its $\mathcal{L}$-variable, one has a map $\mathcal{A} \stackrel{z}{\rightarrow} \mathcal{L}^{\star}$ defined by $\langle z(a), l\rangle=\zeta(a, l)$. One has $\left\langle z_{\star a}(A), l\right\rangle=$ $\xi(\rho(A) \cosh (a) l)=-\Omega(A, \cosh (a) l)$. Proposition 4.4 is now clear regarding the expression of the midpoint map given in Proposition 4.2.

\section{Remarks 4.5.}

(i) This sufficient condition for existence of a global midpoint map is necessary as well, as it will be proven in Section 6 (Corollary 6.3).

(ii) It is easy to determine the $\operatorname{Jacobian} \operatorname{Jac}_{\phi}(a)$, of the twisting diffeomorphism at point $a \in \mathcal{A}$. Indeed, identifying the tangent space $T_{a}(\mathcal{A})$ with $\mathcal{A}$, one has for all $A \in \mathcal{A}$ and $l \in \mathcal{L}$,

$$
\left.\frac{d}{d t}\right|_{0} \Omega(\phi(a+t A), l)=\xi(\rho(A) \cosh (a) l)
$$

that is

$$
\Omega\left(\phi_{\star_{a}}(A), l\right)=\Omega(A, \cosh (a) l) .
$$

Therefore, the linear map $\phi_{\star_{a}}: \mathcal{A} \rightarrow \mathcal{A}$ is "adjoint" to the linear transformation $\left.\cosh (a)\right|_{\mathcal{L}}: \mathcal{L} \rightarrow \mathcal{L}$. Hence

$$
\operatorname{Jac}_{\phi}(a)=\operatorname{det}\left(\left.\cosh (a)\right|_{\mathcal{L}}\right) .
$$

Note that $\left.\cosh (a)\right|_{\mathcal{L}}$ is indeed $\mathcal{L}$-valued since $\rho(a)^{2}(\mathcal{L}) \subset[\mathcal{A}, \mathcal{K}] \subset \mathcal{L}$. Observe also that in the case where $\mathcal{G}$ is nilpotent, one has $\operatorname{Jac}_{\phi}(a)=$ $1 \forall a \in \mathcal{A}$.

Definition 4.6. Let $(\mathcal{G}=\mathcal{B} \oplus \mathcal{A}, \sigma, \Omega)$ be an ESET with splitting homomorphism $\rho: \mathcal{A} \rightarrow \operatorname{End}(\mathcal{B})$. For all $a \in \mathcal{A}$, write

$$
\rho(a)=\rho_{N}(a)+\rho_{S}(a)
$$

for the Jordan-Chevalley decomposition of the (complex linearly extended) endomorphism $\rho(a): \mathcal{B}^{c} \rightarrow \mathcal{B}^{c}$, with $\mathcal{B}^{c}=\mathcal{B} \otimes \mathbb{C}$, and where $\rho_{N}(a)$ (respectively $\rho_{S}(a)$ ) denotes the nilpotent (respectively semisimple) part of the endomorphism $\rho(a)$. The ESET is said to be standard if there exists $a^{N}$ and $a^{S}$ in $\mathcal{A}$ such that

$$
\begin{aligned}
& \rho_{N}(a)=\rho\left(a^{N}\right) \text { and } \\
& \rho_{S}(a)=\rho\left(a^{S}\right) .
\end{aligned}
$$


Proposition 4.7. Every elementary solvable exact triple is a sub-triple of a standard one.

Proof. Let $(\mathcal{G}=\mathcal{B} \oplus \mathcal{A}, \sigma, \Omega)$ denote our starting exact triple. The map $\rho: \mathcal{A} \rightarrow \operatorname{End}(\mathcal{B})$ being injective (because $\Omega$ is nondegenerate), we may identify $\mathcal{A}$ with its image: $\mathcal{A}=\rho(\mathcal{A})$. Let $\Sigma: \operatorname{End}(\mathcal{B}) \rightarrow \operatorname{End}(\mathcal{B})$ be the automorphism induced by the conjugaison with respect to the involution $\left.\sigma\right|_{\mathcal{B}} \in G L(\mathcal{B})$, i.e., $\Sigma=A d\left(\left.\sigma\right|_{\mathcal{B}}\right)$. The automorphism $\Sigma$ is involutive and preserves the canonical Levi decomposition $\operatorname{End}(\mathcal{B})=\mathcal{Z} \oplus \operatorname{sl}(\mathcal{B})$, where $\mathcal{Z}$ denotes the center of $\operatorname{End}(\mathcal{B})$. Now, writing the element $a=\rho(a) \in \mathcal{A}$ as $a=a_{Z}+a_{0}$ with respect to this decomposition, one has: $\Sigma(a)=a_{Z}+$ $\Sigma\left(a_{0}\right)=-a=-a_{Z}-a_{0}$ because the endomorphisms $a$ and $\left.\sigma\right|_{\mathcal{B}}$ anticommute. Hence $\Sigma\left(a_{0}\right)=-2 a_{Z}-a_{0}$ and therefore $a_{Z}=0$. So, $\mathcal{A}$ actually lies in the semisimple part $\operatorname{sl}(\mathcal{B})$. For any $x \in \operatorname{sl}(\mathcal{B})$, we denote by $x=x^{S}+$ $x^{N}, \quad x^{S}, x^{N} \in \operatorname{sl}(\mathcal{B})$ its abstract Jordan-Chevalley decomposition. Observe that, writing $s l(\mathcal{B})=s l_{+} \oplus s l_{-}$for the decomposition in $( \pm 1)-\Sigma$ - eigenspaces, one has : $\mathcal{A} \subset s l_{-}$. Also, $\mathcal{N}:=\left\{a^{N}\right\}_{a \in \mathcal{A}}$ is an Abelian subalgebra in $s l_{-}$ commuting with $\mathcal{A}$. One therefore may define the Abelian subalgebra in $s l_{-}$:

$$
\mathcal{A}^{\prime}=\mathcal{A}+\mathcal{N}
$$

Canonically attached to $\mathcal{A}^{\prime}$, one has the homomorphism: $\rho^{\prime}: \mathcal{A}^{\prime} \rightarrow \operatorname{End}(\mathcal{B})$ which anticommutes with $\left.\sigma\right|_{\mathcal{B}}$, in particular: $\rho^{\prime}(\mathcal{A}) \mathcal{L}=\mathcal{K}$.

Let $\xi \in \mathcal{K}^{\star}$ be the element whose coboundary defines the symplectic structure on $\mathcal{P}=\mathcal{A} \oplus \mathcal{L}$ and denote by $\mathcal{R} \subset \mathcal{A}^{\prime} \oplus \mathcal{L}$ the radical of the coboundary $\delta \xi: \bigwedge^{2}\left(\mathcal{A}^{\prime} \times \rho_{\rho^{\prime}} \mathcal{B}\right) \rightarrow \mathbb{R}$. Observe that $\mathcal{R} \subset \mathcal{A}^{\prime}$. Hence, since $\mathcal{A}^{\prime} \oplus \mathcal{L}=\mathcal{P} \oplus \mathcal{R}$, one has $\mathcal{A}^{\prime}=\mathcal{A} \oplus \mathcal{R}$. At last, let us denote by $\mathcal{Z}(\mathcal{G})$ the center of $\mathcal{G}$.

Case 1: $\mathcal{Z}(\mathcal{G})=0$.

Then $\mathcal{G}$ is a transvection algebra (cf. Remark 2.10). We form $\mathcal{L}^{\prime}=$ $\mathcal{L} \oplus \mathcal{R}^{\star}$, set $\mathcal{B}^{\prime}=\mathcal{K} \oplus \mathcal{L}^{\prime}$, and extend $\rho^{\prime}$ to a homomorphism $\rho^{\prime}: \mathcal{A}^{\prime} \rightarrow$ $\operatorname{End}\left(\mathcal{B}^{\prime}\right)$ as $\rho^{\prime}\left(\mathcal{A}^{\prime}\right) \mathcal{R}^{\star}=0$. This homomorphism anticommutes with the involution $\left.\sigma^{\prime}\right|_{\mathcal{B}^{\prime}}=i d_{\mathcal{K}} \oplus\left(-i d_{\mathcal{L}^{\prime}}\right)$, hence one has an involutive Lie algebra: $\left(\mathcal{A}^{\prime} \times{ }_{\rho^{\prime}} \mathcal{B}^{\prime},\left.\left(-i d_{\mathcal{A}^{\prime}}\right) \oplus \sigma^{\prime}\right|_{\mathcal{B}^{\prime}}\right)$. Now, we define a symplectic structure $\Omega^{\prime}$ on $\mathcal{P}^{\prime}=\mathcal{A}^{\prime} \oplus \mathcal{L}^{\prime}$ by:

$$
\begin{gathered}
\left.\Omega^{\prime}\right|_{\mathcal{P} \times \mathcal{P}}=\left.\Omega\right|_{\mathcal{P} \times \mathcal{P}} \\
\left.\Omega^{\prime}\right|_{\mathcal{P} \times\left(\mathcal{R} \oplus \mathcal{R}^{\star}\right)}=0 \\
\Omega^{\prime}\left(r^{*}, r\right)=\left\langle r^{*}, r\right\rangle \quad \forall r \in \mathcal{R} ; r^{*} \in \mathcal{R}^{\star} .
\end{gathered}
$$


The symplectic structure $\Omega^{\prime}$ turns out to be $\mathcal{K}$-invariant. Indeed, one has: $\left[\mathcal{K}, \mathcal{R}^{\star}\right]=\left[[\mathcal{A}, \mathcal{L}], \mathcal{R}^{\star}\right]=0$ (by Jacobi) and $[\mathcal{K}, \mathcal{R}] \subset \mathcal{L}$. Now, considering a one-dimensional central extension of $\mathcal{A}^{\prime} \times{ }_{\rho^{\prime}} \mathcal{B}^{\prime}$, one gets the desired standard exact triple.

Case 2: $\mathcal{Z}(\mathcal{G}) \neq 0$.

Define $E \in \mathcal{Z}(\mathcal{G}) \subset \mathcal{K}$ by $\xi(E)=1$. Set $\mathcal{L}^{\prime}=\mathcal{L} \oplus \mathcal{R}^{\star}, \mathcal{B}^{\prime}=\mathcal{K} \oplus \mathcal{L}^{\prime}$, and endow the vector space $\mathcal{G}^{\prime}=\mathcal{B}^{\prime} \oplus \mathcal{A}^{\prime}$ with the skewsymmetric bracket defined by:

$$
\begin{gathered}
{\left.[,]_{\mathcal{G}^{\prime}}\right|_{\mathcal{G} \times \mathcal{G}}=[,]_{\mathcal{G}}} \\
{\left[r^{*}, r\right]=\left\langle r^{*}, r\right\rangle E \quad \forall r \in \mathcal{R} ; r^{*} \in \mathcal{R}^{\star}} \\
{[r, b]=\rho^{\prime}(r) b \quad \forall r \in \mathcal{R} ; b \in \mathcal{B} \subset \mathcal{B}^{\prime},}
\end{gathered}
$$

the other brackets being zero. Endowed with this structure $\mathcal{G}^{\prime}$ turns out to be a Lie algebra. Moreover the coboundary $\Omega^{\prime}=\delta \xi: \bigwedge^{2}\left(\mathcal{G}^{\prime}\right) \rightarrow \mathbb{R}$ restricts to $\mathcal{P}^{\prime}=\mathcal{A}^{\prime} \oplus \mathcal{L}^{\prime}$ as a symplectic structure. Therefore the triple $\left(\mathcal{G}^{\prime}, i d_{\mathcal{K}} \oplus\left(-i d_{\mathcal{P}^{\prime}}\right), \Omega^{\prime}\right)$ is the desired standard exact triple.

Remark 4.8. Observe that the exact triples constructed in Cases 1 and 2 above are both such that

$$
\operatorname{dim} \mathcal{Z}(\mathcal{G})=1
$$

This ensures they are one-dimensional central extensions of (transvection) symplectic triples.

Led by the example in the preceding section, one observes

Proposition 4.9. Let $(M=\mathcal{A} \times \mathcal{L}, \omega, s)$ be an elementary solvable symplectic symmetric space. Then, the function

$$
\tilde{u}: M \times M \rightarrow \mathcal{K}
$$

defined by

$$
\tilde{u}\left((a, l),\left(a^{\prime}, l^{\prime}\right)\right)=\sinh \left(a^{\prime}\right) l-\sinh (a) l^{\prime}
$$

satisfies (locally)

$$
\tilde{u}(x, y)=-\tilde{u}(y, x)=-\tilde{u}\left(x, s_{x}(y)\right)=-\tilde{u}\left(x, s_{\frac{x}{2}}(y)\right) \quad \forall x, y \in M
$$

and is $\exp (\mathcal{K})$-invariant. In particular, for every choice of an element $\xi \in$ $\mathcal{K}^{\star}$, the function

$$
u=\xi \circ \tilde{u}
$$


is an admissible two-point function on $M$ (Definition 3.4) provided the midpoint map is globally defined. The associated admissible three-point function (Proposition 3.3) has the form:

$$
S\left(\left(a_{1}, l_{1}\right),\left(a_{2}, l_{2}\right),\left(a_{3}, l_{3}\right)\right)=\xi\left(\oint_{1,2,3} \sinh \left(a_{1}-a_{2}\right) l_{3}\right)
$$

where $\oint_{1,2,3}$ stands for the cyclic summation.

One sees here why it is important to deal with an exact triple rather than the transvection triple. Indeed, one would need, while proceeding a stationary phase method for the deformed product (cf. Dirac's condition in definition 1.5), to obtain the Poisson bracket associated to the the symplectic structure $\omega$ as the first order expansion term. The critical point analysis tells us that the above expression of $S$ yields a first order term equal to $\delta \xi$.

\section{Obtaining the oscillating kernel via star representation theoretical methods.}

In this section, we present a heuristic way to derive oscillatory integral formulae for our strict deformation quantizations (see Theorem 6.13). For the sake of simplicity, we will only treat the case of the two-dimensional example $\left(\mathbb{R}^{2}, \omega^{0}, s\right)$, already mentioned in Example 3.5. The general case will be treated later. The proof of Theorem 6.13 does not depend on the present section, but we believe that it clarifies the "mysterious" formula (16) of the deformed product. This section, together with Section 1, suggests that our construction should generalize to a much wider situation than solvable symmetric space.

Following a classical result of Kostant, the symplectic homogeneous space $(M=G / K, \omega)$ is an equivariant symplectic covering of a coadjoint orbit in $\mathcal{G}^{\star}$. From Proposition 4.2 , one gets a global section $\gamma: \mathcal{P}=\mathcal{A} \times \mathcal{L} \rightarrow$ $G=\mathcal{A} \times \mathcal{K} \times \mathcal{L}$. This yields a global Darboux chart on the coadjoint orbit $\mathcal{O}=A d^{\star}(G) \xi \subset \mathcal{G}^{\star}:$

$$
\mathcal{P} \rightarrow \mathcal{O}: x \rightarrow A d^{\star}(\gamma(x)) \xi
$$

In particular, the Hamiltonian function $\lambda_{X} \in C^{\infty}(\mathcal{P})$ associated to the infinitesimal action of $X \in \mathcal{G}$ (see Section 2) is given by:

$$
\begin{aligned}
\lambda_{X}(x) & =\langle A d(\gamma(x)) \xi, X\rangle \\
& =\left\langle\xi, A d\left(\gamma(x)^{-1}\right) X\right\rangle \\
& =\left\langle\xi, \rho\left(X_{\mathcal{A}}\right) l-e^{-\rho(a)} X_{\mathcal{B}}\right\rangle
\end{aligned}
$$


where $x=(a, l)$ and $X=X_{\mathcal{A}}+X_{\mathcal{B}} \in \mathcal{G}=\mathcal{A} \times \mathcal{B}$. From this last expression, one obtains for $X \in \mathcal{P}$ :

$$
\begin{aligned}
\partial_{l}^{\beta} \lambda_{X} & =0 \quad(\beta \geq 2) \\
\partial_{a}^{\alpha} \partial_{l}^{\beta} \lambda_{X} & =0 \quad(\alpha, \beta \geq 1) \\
\partial_{l} \lambda_{X}(x) & =\left\langle\xi, \rho\left(X_{\mathcal{A}}\right) \partial_{l}\right\rangle \\
\partial_{a}^{\alpha} \lambda_{X}(x) & =\cosh (a)\left\langle\xi, \rho\left(\partial_{a}\right)^{\alpha} X\right\rangle \quad(\alpha \text { odd }, \alpha \geq 1),
\end{aligned}
$$

where $\partial_{a}$ (respectively $\partial_{l}$ ) is an element of $\mathcal{A}$ (respectively $\mathcal{L}$ ) thought of as a constant vector field on $\mathcal{P}$. In particular, if $\star_{\nu}^{M}$ denotes the standard Moyal star product on $(\mathcal{P}, \Omega)$ (cf. formula (2) in Section 1 ), one gets

$$
\left[\lambda_{X}, \lambda_{Y}\right]_{\star_{\nu}^{M}} \stackrel{\text { def. }}{=} \lambda_{X} \star_{\nu}^{M} \lambda_{Y}-\lambda_{Y} \star_{\nu}^{M} \lambda_{X}=2 \nu\left\{\lambda_{X}, \lambda_{Y}\right\} \quad \forall X, Y \in \mathcal{G} .
$$

One refers to this last property as the $\mathcal{G}$-covariance of the Moyal star product $([1])$. The covariance property allows us to define a representation of $\mathcal{G}$ on the space $C^{\infty}(\mathcal{O})[[\nu]]$ of formal power series in the parameter $\nu$ with coefficients in $C^{\infty}(\mathcal{O})$ :

$$
\begin{array}{r}
\rho_{\nu}: \mathcal{G} \rightarrow \operatorname{End}\left(C^{\infty}(\mathcal{O})[[\nu]]\right): \\
\rho_{\nu}(X) u=\frac{1}{2 \nu}\left[\lambda_{X}, u\right]_{\star_{\nu}^{M}} .
\end{array}
$$

Formula (2) in Section 1 yields, using (8),

$$
\begin{aligned}
& \left(\rho_{\nu}(X) u\right)(x) \\
& =\left\{\lambda_{X}, u\right\}-\frac{1}{\nu} \sum_{k=1}^{\infty} \frac{\nu^{2 k+1}}{(2 k+1) !} \cosh (a)\left\langle\xi, \rho\left(\partial_{a}\right)^{2 k+1} X\right\rangle \partial_{l}^{2 k+1} u \\
& =\left\langle\xi, \rho\left(X_{\mathcal{A}}\right) \partial_{l}\right\rangle \partial_{a} u-\frac{\cosh (a)}{\nu} \sum_{k=0}^{\infty} \frac{\nu^{2 k+1}}{(2 k+1) !}\left\langle\xi, \rho\left(\partial_{a}\right)^{2 k+1} X\right\rangle \partial_{l}^{2 k+1} u
\end{aligned}
$$

A partial Fourier transform in the $\mathcal{L}$-variables allows to interpret $\rho_{\nu}$ as a "multiplicative representation". Indeed, setting

$$
F(u)(a, \alpha)=\hat{u}(a, \alpha)=\int_{\mathcal{L}} e^{-i \Omega(\alpha, l)} u(a, l) d l,
$$

one gets:

$$
\begin{aligned}
& \hat{\rho}_{\nu}(X) . \hat{u} \stackrel{\text { def. }}{=} F\left(\rho_{\nu}(X) u\right) \\
& =X_{\mathcal{A}} \cdot \hat{u}-\frac{\cosh (a)}{\nu} \sum_{k=0}^{\infty} \frac{(i \alpha \nu)^{2 k+1}}{(2 k+1) !}\left\langle\xi, \rho\left(\partial_{a}\right)^{2 k+1} X\right\rangle \hat{u}
\end{aligned}
$$


for all $X=X_{\mathcal{A}}+X_{\mathcal{L}} \in \mathcal{P}$. That is, setting $\nu=\frac{\hbar}{2 i}$, for all $X \in \mathcal{P}$ :

$$
\hat{\rho}_{\frac{\hbar}{2 i}}(X) \cdot \hat{u}=X_{\mathcal{A}} \cdot \hat{u}-\frac{2 i \cosh (a)}{\hbar}\left\langle\xi, \sinh \left(\frac{\hbar \alpha}{2}\right) X\right\rangle \hat{u}
$$

or

$$
\hat{\rho}_{\frac{\hbar}{2 i}}(X) \cdot \hat{u}=X_{\mathcal{A}} \cdot \hat{u}+c_{\hbar}(X) \hat{u}
$$

with $c_{\hbar}: \mathcal{P} \rightarrow C^{\infty}\left(\mathbb{R}^{2}\right)$ defined by

$$
c_{\hbar}(X)(a, \alpha) \stackrel{\text { def. }}{=}-\frac{2 i \cosh (a)}{\hbar}\left\langle\xi, \sinh \left(\frac{\hbar \alpha}{2}\right) X\right\rangle .
$$

The expression of the cocycle $c_{\hbar}$ is very similar to the one of the "twisting map" $\zeta$ associated to the three-point function $S$ (cf. Proposition 4.4). From this observation, we now derive a commutative multiplicative law on the functions on $\mathbb{R}^{2}=\{(a, \alpha)\}$ for which representation $\hat{\rho}$ acts by derivations. We start by observing that, for all $\partial_{l} \in \mathcal{L}$, one has $\partial_{l} \zeta(\alpha, l)=\left\langle\xi, \sinh (\alpha) \partial_{l}\right\rangle$. Hence, setting

$$
\zeta_{\hbar}(\alpha, l) \stackrel{\text { def. }}{=} \frac{2}{\hbar} \zeta\left(\frac{\hbar}{2} \alpha, l\right)
$$

and

$$
\mu=-\frac{1}{i \cosh (a)},
$$

one gets $\partial_{l} \zeta_{\hbar}(\alpha, l)=\mu c_{\hbar}\left(\partial_{l}\right)(a, \alpha)$. Therefore, defining formally

$$
\left(\mathcal{Z}_{\hbar} u\right)(a, \alpha) \stackrel{\text { def. }}{=} \int_{\mathcal{L}} e^{-i \zeta_{\hbar}(\alpha, l)} u(a, l) d l
$$

for (reasonable) $u \in C^{\infty}(\mathcal{P})$, an integration by parts argument leads us to $\mathcal{Z}_{\hbar}\left(\partial_{l} u\right)=i \mu c_{\hbar}\left(\partial_{l}\right) \mathcal{Z}_{\hbar}(u)$ or

$$
\mathcal{Z}_{\hbar}^{-1}\left(c_{\hbar}\left(\partial_{l}\right) f\right)=\frac{1}{i \mu} \partial_{l} \mathcal{Z}_{\hbar}^{-1}(f)
$$

whenever this last formula makes sense for $f \in C^{\infty}\left(\mathbb{R}^{2}\right)$. When well-defined, the following (commutative) product

$$
f \bullet \hbar \stackrel{\text { def. }}{=} \mathcal{Z}_{\hbar}\left(\mathcal{Z}_{\hbar}^{-1} f . \mathcal{Z}_{\hbar}^{-1} g\right)
$$

is then invariant under the representation $\hat{\rho}$. Indeed, since the integral in the definition of $\mathcal{Z}_{\hbar}$ is only taken on the $\mathcal{L}$-variable, the product $\bullet_{\hbar}$ is invariant under $\mathcal{A}$ (observe that $c_{\hbar}(\mathcal{A})=0$ ). The algebra $\mathcal{G}$ being generated by $\mathcal{P}$, it 
is therefore sufficient to prove $c_{\hbar}\left(\partial_{l}\right) f \bullet_{\hbar} g=\left(c_{\hbar}\left(\partial_{l}\right) f\right) \bullet_{\hbar} g+f \bullet_{\hbar}\left(c_{\hbar}\left(\partial_{l}\right) g\right)$. The RHS is actually $\frac{1}{i \mu} \mathcal{Z}_{\hbar}\left(\left(\partial_{l} \mathcal{Z}_{\hbar}^{-1} f\right) \cdot \mathcal{Z}_{\hbar}^{-1} g+\mathcal{Z}_{\hbar}^{-1} f \cdot \partial_{l} \mathcal{Z}_{\hbar}^{-1} g\right)$ using (9) and the fact that multiplication by $\mu$ commutes with the transformation $\mathcal{Z}_{\hbar}$. One therefore has $\frac{1}{i \mu} \mathcal{Z}_{\hbar} \partial_{l}\left(\mathcal{Z}_{\hbar}^{-1} f . \mathcal{Z}_{\hbar}^{-1} g\right)$ or $\frac{1}{i \mu} \mathcal{Z}_{\hbar} \partial_{l} \mathcal{Z}_{\hbar}^{-1}\left(f \bullet_{\hbar} g\right)$ which equals $c_{\hbar}\left(\partial_{l}\right) f \bullet_{\hbar} g$ using $(9)$ again.

If one interprets the commutative product $\bullet \hbar$ as the underlying product to the algebra of functions on a commutative $\hbar$-dependent manifold, say $M_{\hbar}$, its invariance under $\hat{\rho}$ tells us that $\mathcal{G}$ is realized via $\hat{\rho}$ as a subalgebra of tangent vector fields over $M_{\hbar}$. It turns out that this action of $\mathcal{G}$ on $M_{\hbar}$ is equivalent to the one of $\mathcal{G}$ on $M$. Indeed for all $X \in \mathcal{G}$, one has:

$$
\mathcal{Z}_{\hbar}^{-1} \circ \hat{\rho}_{\frac{\hbar}{2 i}}(X) \circ \mathcal{Z}_{\hbar}=X^{\star}
$$

Again, it is sufficient to prove this last formula only for $X=\partial_{l} \in \mathcal{L}$. In this case, formula (9) identifies the LHS action on $u \in C^{\infty}(\mathcal{P})$ to $\frac{1}{i \mu} \partial_{l} u$ which is precisely $\partial_{l}^{\star} u$.

Formula (10) leads us to consider the transformation $T_{\hbar}$ defined by

$$
T_{\hbar}=F^{-1} \circ \mathcal{Z}_{\hbar}
$$

which intertwines our $\star$-representation $\hat{\rho}_{\frac{\hbar}{2 i}}$ with the infinitesimal (coadjoint) action of the transvection algebra. In other words, the formal product on $C^{\infty}(\mathcal{P})[[\hbar]]$ defined by

$$
u \star \hbar v \stackrel{\text { def. }}{=} T_{\hbar}^{-1}\left(T_{\hbar} u \star_{\frac{\hbar}{2 i}}^{M} T_{\hbar} v\right)
$$

is invariant under the coadjoint action of $G$ on $\mathcal{O}=\mathcal{P}$.

\section{WKB-Quantization.}

Proposition 6.1. Let $(M, \omega, s)$ be an elementary solvable symplectic symmetric space. Let $\phi: \mathcal{A} \rightarrow \mathcal{A}$ be the associated twisting map (cf. Definition 4.3). Assume that $(M, \omega, s)$ admits a globally defined midpoint map (cf. Proposition 4.2 (ii)). Let $\|$,$\| be a norm on the vector space \mathcal{A}$. Then

$$
\liminf _{a \rightarrow \infty} \frac{\|\phi(a)\|}{\|a\|}>0 \text {. }
$$

Before passing to the proof, we observe

Lemma 6.2. Let $V$ be a finite dimensional real vector space. Let $\mathcal{A}$ be a non trivial Abelian subalgebra of $\operatorname{End}(V)$ such that 
(i) if the Jordan-Chevalley decomposition (cf. Definition 4.6) of a $\in \mathcal{A}$ writes: $a=a^{S}+a^{N}$ then

$$
a^{S}, a^{N} \in \mathcal{A}
$$

(ii) every non zero (complex) eigenvalue of $a^{S}$ has a non trivial real part.

Consider the function $\mathcal{A} \rightarrow \operatorname{End}(V): A \rightarrow \sinh (a)$. Then,

$$
\liminf _{a \rightarrow \infty} \frac{\|\sinh (a)\|_{o p}}{\|a\|_{o p}}>0
$$

where $\|,\|_{o p}$ denotes the operator norm on $\operatorname{End}(V)$ defined with respect to any norm compatible with the vector space structure on $V$.

Proof. Let us first extend the action of $\mathcal{A}$ on $V \mathbb{C}$-linearly to a (complex) action of $\mathcal{A}$ on $V^{c}=V \otimes \mathbb{C}$. Denote by $V^{c}=\oplus_{\lambda \in \Phi} V_{\lambda}$ the weight space decomposition with respect to the Abelian subalgebra of semisimple elements: $\mathcal{S}=\left\{a^{S}\right\}_{a \in \mathcal{A}}$, where $\Phi$ denotes the set of weights of the action of $\mathcal{S}$. Observe that, setting $\mathcal{N}=\left\{a^{N}\right\}_{a \in \mathcal{A}}$, one has $\mathcal{N} . V_{\lambda} \subset V_{\lambda}$ for all $\lambda$ in $\Phi$. In each $V_{\lambda}$, fix a basis $e_{\lambda}$ for which the matrix associated to any $n \in \mathcal{N}$ is upper triangular. In particular, in the basis $e=\left\{e_{\lambda}\right\}_{\lambda \in \Phi}$, the matrix associated to any $a \in \mathcal{A}$ is upper triangular with elements $\lambda\left(a^{S}\right)$ on the principal diagonal. Let $\tilde{\mathcal{A}}$ be a maximal Abelian subalgebra of $\operatorname{End}\left(V^{c}\right)$ which contains $\mathcal{A}$. Maximality implies that, similarly to $\mathcal{A}$, one has a decomposition in semisimple and nilpotent parts: $\tilde{\mathcal{A}}=\tilde{\mathcal{S}} \oplus \tilde{\mathcal{N}}$. Note that $\sinh (\mathcal{A}) \subset \sinh (\tilde{\mathcal{A}}) \subset \tilde{\mathcal{A}}$.

On $\tilde{\mathcal{A}}$, we consider the norm:

$$
\|\tilde{a}=\tilde{s}+\tilde{n}\| \stackrel{\text { def. }}{=} \max \left\{\|\tilde{s}\|_{o p},\|\tilde{n}\|_{o p}\right\} \quad(\tilde{s} \in \tilde{\mathcal{S}}, \tilde{n} \in \tilde{\mathcal{N}})
$$

where \|\|$_{o p}$ denotes the operator norm on $\operatorname{End}\left(V^{c}\right)$ associated to the choice of a norm on $V^{c}$. Now, we observe that for all sequences $\left\{s_{k}\right\}_{k \in \mathbb{N}} \subset \mathcal{S}$, one has

$$
\lim _{k \rightarrow \infty} \frac{\left\|\sinh \left(s_{k}\right)\right\|}{\left\|s_{k}\right\|} \neq 0 .
$$

The proof of the last assertion is divided into the three following steps.

First, for any fixed $k \in \mathbb{N}$, one can find a weight $\lambda^{(k)} \in \Phi$ which realizes $\left|\lambda^{(k)}\left(s_{k}\right)\right|=\left\|s_{k}\right\|_{o p}$. In particular,

$$
\frac{\left\|\sinh \left(s_{k}\right)\right\|}{\left\|s_{k}\right\|} \geq \frac{\left|\sinh \left(\lambda^{(k)}\left(s_{k}\right)\right)\right|}{\left|\lambda^{(k)}\left(s_{k}\right)\right|} .
$$


Assume by the absurd that

$$
\lim _{k \rightarrow \infty} \frac{\left\|\sinh \left(s_{k}\right)\right\|}{\left\|s_{k}\right\|}=0
$$

Since $\Phi$ is a finite set, one can then find a weight $\lambda \in \Phi$ and a partial sequence $\left\{s_{l}^{\prime}\right\} \subset\left\{s_{k}\right\}$ such that

$$
\lim _{l \rightarrow \infty} \frac{\left|\sinh \left(\lambda\left(s_{l}^{\prime}\right)\right)\right|}{\left|\lambda\left(s_{l}^{\prime}\right)\right|}=0
$$

Secondly, observe that every weight $\lambda \in \Phi$ writes $\lambda=\alpha_{\lambda}+i \beta_{\lambda}$ with $\alpha_{\lambda}$ and $\beta_{\lambda}$ in the real vector space $\mathcal{S}^{\star}$ with the property that if $s \in \operatorname{ker} \alpha_{\lambda}$ then $\lambda(s)=\beta_{\lambda}(s)=0$ (hypothesis (ii)). Hence $\operatorname{ker} \alpha_{\lambda} \subset \operatorname{ker} \beta_{\lambda}$, and there exists a real $t_{\lambda} \in \mathbb{R}$ such that $\lambda=\left(1+i t_{\lambda}\right) \alpha_{\lambda}$.

Thirdly, for all $z=x+i y \in \mathbb{C}$, one has $|\sinh (z)|^{2}=\sinh (x)^{2}+\sin (y)^{2}$. Hence writing $\lambda\left(s_{l}^{\prime}\right)=x_{l}+i y_{l}$, the relation (11) yields

$$
\lim _{l \rightarrow \infty} \frac{\sinh \left(x_{l}^{2}\right)}{x_{l}^{2}+y_{l}^{2}}=0
$$

But, by our preceding observation, there exists a real number $t$ such that $y_{l}=t x_{l}$. Hence $\left|\lambda\left(s_{\lambda}^{\prime}\right)\right|^{2}=\left(1+t^{2}\right) x_{l}^{2}$. This, combined with (12), yields a real sequence $x_{l}$ such that $\lim _{l \rightarrow \infty} \frac{\sinh \left(x_{l}^{2}\right)}{x_{l}^{2}}=0$, and we reach a contradiction.

Suppose, that $\left\{a_{k}\right\}_{k \in \mathbb{N}}$ is a sequence of elements in $\mathcal{A}$ for which the sequence $\left\|a_{k}\right\|$ diverges to infinity and such that $\lim _{k \rightarrow \infty} \frac{\left\|\sinh \left(a_{k}\right)\right\|_{o p}}{\left\|a_{k}\right\| \|_{o p}}=0$. Every two norms on $\mathcal{A}$ being equivalent, one has: $\lim _{k \rightarrow \infty} \frac{\left\|\sinh \left(a_{k}\right)\right\|}{\left\|a_{k}\right\|}=0$. Consider the following sequences partitioning $\left\{a_{k}\right\}$ :

$$
\begin{aligned}
& \left\{\nu_{p}\right\}=\left\{a_{k}=a_{k}^{S}+a_{k}^{N} \text { such that }\left\|a_{k}^{N}\right\|>\left\|a_{k}^{S}\right\|\right\} \\
& \left\{\sigma_{q}\right\}=\left\{a_{k}=a_{k}^{S}+a_{k}^{N} \text { such that }\left\|a_{k}^{N}\right\| \leq\left\|a_{k}^{S}\right\|\right\}
\end{aligned}
$$

One has $\lim _{q \rightarrow \infty} \frac{\left\|\sinh \left(\sigma_{q}\right)\right\|}{\left\|\sigma_{q}\right\|}=0$. And, since

$$
\begin{aligned}
\frac{\left\|\sinh \left(\sigma_{q}\right)\right\|}{\left\|\sigma_{q}\right\|} & =\frac{\max \left\{\left\|\left(\sinh \left(\sigma_{q}\right)\right)^{S}\right\|,\left\|\left(\sinh \left(\sigma_{q}\right)\right)^{N}\right\|\right\}}{\left\|\sigma_{q}^{S}\right\|} \\
& \geq \frac{\left\|\left(\sinh \left(\sigma_{q}\right)\right)^{S}\right\|}{\left\|\sigma_{q}^{S}\right\|}=\frac{\left\|\sinh \left(\sigma_{q}^{S}\right)\right\|}{\left\|\sigma_{q}^{S}\right\|}
\end{aligned}
$$


our previous observation implies that $\left\{\sigma_{q}\right\}=\emptyset$, i.e., that $\left\{a_{k}\right\}=\left\{\nu_{p}\right\}$. As above, one gets $\lim _{k} \frac{\left\|\left(\sinh \left(a_{k}\right)\right)^{N}\right\|}{\left\|a_{k}^{N}\right\|}=0$, that is,

$$
\lim _{k \rightarrow \infty} \frac{1}{\left\|a_{k}^{N}\right\|}\left\{\cosh \left(a_{k}^{S}\right) \sinh \left(a_{k}^{N}\right)+\sinh \left(a_{k}^{S}\right)\left(\cosh \left(a_{k}^{N}\right)-\mathbf{1}\right)\right\}=0 .
$$

For all $a \in \mathcal{A}$, the endomorphisms $\sinh \left(a^{N}\right)$ and $\cosh \left(a^{N}\right)-\mathbf{1}$ are linearly independent in $\operatorname{End}\left(V^{c}\right)$. Indeed, those are sums of powers of the upper triangular nilpotent matrix $a^{N}$. Hence, none of the terms of $\cosh \left(a^{N}\right)-\mathbf{1}$ can cancel the term $a^{N}$ in $\sinh \left(a^{N}\right)$. Therefore, since $\cosh \left(a^{S}\right)$ as well as $\sinh \left(a^{S}\right)$ act diagonally, the terms occurring in the bracket of expression (13) are linearly independent. In particular, $\lim _{k} \cosh \left(a_{k}^{S}\right) \frac{1}{\left\|a_{k}^{N}\right\|} \sinh \left(a_{k}^{N}\right)=0$. Therefore, $\cosh \left(a_{k}^{S}\right)$ being invertible for all $k$, one gets

$$
\lim _{k} \frac{1}{\left\|a_{k}^{N}\right\|} \sinh \left(a_{k}^{N}\right)=0 .
$$

Again, since the matrix $a_{k}^{N}$ is upper triangular, one gets $\lim _{k} \frac{1}{\left\|a_{k}^{N}\right\|} a_{k}^{N}=0$, a contradiction.

Proof of Proposition 6.1. Proposition 4.7 reduces to prove the assertion only for standard ESET's. Indeed, the twisting map associated to any ESET can be realized as the restriction of the twisting map of a standard ESET to a linear subspace. We therefore assume our $\operatorname{ESET}(\mathcal{G}, \sigma, \Omega)$ to be standard.

Let us define $C_{s l_{-}}(\mathcal{A})$ to be the intersection of the centralizer algebra of $\mathcal{A}=\rho(\mathcal{A})$ in $\operatorname{End}(\mathcal{B})$ and $s l_{-}$(cf. Proof of Proposition 4.7). For $X \in$ $C_{s l_{-}}(\mathcal{A})$, we set

$$
\|X\| \stackrel{\text { def. }}{=} \sup _{\|l\| \leq 1}\{|\xi(X . l)|\}
$$

where $l \in \mathcal{L}=\mathcal{B} \cap \mathcal{P}$ and $\delta \xi=\Omega \quad\left(\xi \in \mathcal{K}^{\star}\right)$. This defines a norm on $C_{s l_{-}}(\mathcal{A})$. Indeed, if $X \in C_{s l_{-}}(\mathcal{A})$ is such that $\xi(X . \mathcal{L})=0$, one has $\xi(X .[\mathcal{A}, \mathcal{K}])=$ $\xi[\mathcal{A}, X . \mathcal{K}]=\Omega(\mathcal{A}, X . \mathcal{K})=0$, hence $X . \mathcal{K}=0$. Also, $0=X . \mathcal{K}=X$. $[\mathcal{A}, \mathcal{L}]=$ $[\mathcal{A}, X . \mathcal{L}]$, hence $X . \mathcal{L} \subset \mathcal{Z}(\mathcal{G})$. By item (ii) of Remark 2.10, one has either $X . \mathcal{L}=0$ or $X . \mathcal{L} \subset \mathbb{R} . E$ with $\xi(E)=1$, which implies $X . \mathcal{L}=0$ too since one assumed $\xi(X . \mathcal{L})=0$. Now, observe that $\sinh (\mathcal{A}) \subset C_{s l_{-}}(\mathcal{A})$, and, that for all $a \in \mathcal{A}$, one has:

$$
\|\sinh (a)\|=\sup _{\|l\| \leq 1}\{|\xi(\sinh (a) l)|\}=\sup _{\|l\| \leq 1}\{|\Omega(\phi(a), l)|\}=\|\phi(a)\| .
$$


Therefore, the norms $\|,\|_{o p}$ and $\|$,$\| being equivalent, Lemma 6.2$ implies Proposition 6.1.

We now have the following geometric property.

Corollary 6.3. Let $(M, \omega, s)$ be an elementary solvable symplectic symmetric space. Then $(M, \omega, s)$ admits a globally defined midpoint map $x \mapsto \frac{x}{2}$ (if and) only if the twisting map $\phi: \mathcal{A} \rightarrow \mathcal{A}$ (cf. Definition 4.3) is a global diffeomorphism.

Proof. With the same notations as in the proof of Proposition 4.4, one has a map $\phi: \mathcal{A} \rightarrow \mathcal{A}$ defined by $\phi=\Omega \circ z$. The argument used in the same proof tells us that $\phi$ is a local diffeomorphism. Now, Proposition 6.1 implies that the map $\phi: \mathcal{A} \rightarrow \mathcal{A}$ is proper. Indeed, $\phi$ being continuous, one just needs to show that the inverse image of a ball is bounded. So, let $\left\{x_{k}\right\} \subset \mathcal{A}$ be such that

$$
\left\|\phi\left(x_{k}\right)\right\|<R .
$$

Since $0<c<\liminf \frac{\left\|\phi\left(x_{k}\right)\right\|}{\left\|x_{k}\right\|} \leq \frac{R}{\left\|x_{k}\right\|}$, one gets $\left\|x_{k}\right\| \leq \frac{R}{c}$ as soon as $k$ is large enough.

Therefore, nonetheless the map $\phi$ is open it is also closed, hence surjective. The twisting map is therefore a covering map. Since the fundamental group of $\mathcal{A}$ is trivial, it is a diffeomorphism.

Notations 6.4. Let $V$ be a finite dimensional real vector space. We denote by $\mathcal{S}(V)$ the space of Schwartz (complex-valued) functions on $V$. Accordingly, $\mathcal{S}^{\prime}(V)$ stands for the space of tempered distributions on $V$.

Proposition 6.5. Let $(M, \omega, s)$ be an elementary solvable symplectic symmetric space, with associated ESET $(\mathcal{G}=\mathcal{A} \oplus \mathcal{B}, \sigma, \Omega)$ (cf. Definition 4.1). Assume that $(M, \omega, s)$ admits a globally defined midpoint map. Let $\phi: \mathcal{A} \rightarrow$ $\mathcal{A}$ be the associated twisting diffeomorphism (cf. Corollary 6.3). Then, one has

(i) $\phi^{\star} \mathcal{S}(\mathcal{A}) \subset \mathcal{S}(\mathcal{A})$ and

(ii) $\left(\phi^{-1}\right)^{\star} \mathcal{S}(\mathcal{A}) \subset \mathcal{S}^{\prime}(\mathcal{A})$.

We use the following lemmas. 
Lemma 6.6. Within the hypotheses and notations of Lemma 6.2, the function

$$
\mathcal{A} \rightarrow \operatorname{End}(V): a \rightarrow(\cosh (a))^{-1}
$$

is well-defined as a tempered analytic function from $\mathcal{A}$ to the (real Banach) space $\operatorname{End}(V)$. The same holds for $a \rightarrow \tanh (a)$.

Proof. One has $\cosh (a)=\cosh \left(a^{S}+a^{N}\right)=\cosh \left(a^{S}\right)\left(\cosh \left(a^{N}\right)+\right.$ $\left.\tanh \left(a^{S}\right) \sinh \left(a^{N}\right)\right)$. In the basis $e$ considered in the proof of Lemma 6.2, $\cosh \left(a^{S}\right)$ and $\tanh \left(a^{S}\right)$ are diagonal matrices whose elements are in $\left\{\cosh \left(\lambda\left(a^{S}\right)\right), \tanh \left(\lambda\left(a^{S}\right)\right)\right\}_{\lambda \in \Phi}$. Since each weight is of the form $\lambda=(1+i t) \alpha$ (cf. Proof of Lemma 6.2), $\cosh \left(a^{S}\right)^{-1}$ is a Schwartz function, and $\tanh \left(a^{S}\right)$ together with all its derivatives are bounded.

The factor $\cosh \left(a^{N}\right)+\tanh \left(a^{S}\right) \sinh \left(a^{N}\right)$ is an upper triangular matrix whose diagonal elements are equal to 1 . Moreover, it is a polynomial in the variable $a^{N}$. Therefore its inverse is a tempered function in the variable $a$. A similar argument (but simpler) yields the second part of the assertion.

Lemma 6.7. With the hypotheses of Proposition 6.1, one has:

$$
\sinh \left(\phi^{-1}(a)\right)=\rho(a)
$$

for all $a \in \mathcal{A}$.

Proof. One has, for all $k \in \mathcal{K}$ and $a, A \in \mathcal{A}$ :

$$
\begin{gathered}
\Omega\left(\sinh \left(\phi^{-1}(a)\right) k, A\right)=\xi\left[\sinh \left(\phi^{-1}(a)\right) k, A\right]=\xi\left(\sinh \left(\phi^{-1}(a)\right)[k, A]\right)= \\
\zeta\left(\phi^{-1}(a),[k, A]\right)=\Omega\left(\phi\left(\phi^{-1}(a)\right),[k, A]\right)=\Omega(a,[k, A])=\Omega([a, k], A) .
\end{gathered}
$$

Hence $\sinh \left(\phi^{-1}(a)\right) k=[a, k]$. Moreover, $\sinh \left(\phi^{-1}(a)\right)[k, A]=$ $\left[\sinh \left(\phi^{-1}(a)\right) k, A\right]=[[a, k], A]=[a,[k, A]]$.

Lemma 6.8. Let $\mathcal{B}(E)$ be the space of bounded linear operators on the Banach space $E$. Let $a \in \mathcal{B}(E)$ be invertible and such that $\left\|a^{-1}\right\| \leq 1$. Then, $\left\|a^{2}\right\| \geq\|a\|$.

Proof. One has $\sup _{x \in E,\|x\| \leq 1}\left\{\left\|a^{2} a^{-1} x\right\|\right\} \leq \sup _{y \in E,\|y\| \leq\left\|a^{-1}\right\|}\left\{\left\|a^{2} y\right\|\right\} \leq$ $\sup _{x \in E,\|x\| \leq 1}\left\{\left\|a^{2} x\right\|\right\}$. 
Proof of Proposition 6.5. As in Proposition 6.1, we can assume our ESET to be standard. A function $u \in C^{\infty}(\mathcal{A})$ is Schwartz if and only if, for all multiindex $\alpha$, and all positive integer $N$, one has $\sup _{a \in \mathcal{A}}\left\{\|a\|^{N}\left|\left(D^{\alpha} u\right)(a)\right|\right\}<\infty$. Consider $u \in \mathcal{S}(\mathcal{A})$. Then, one has

$$
\sup _{a \in \mathcal{A}}\left\{\|a\|^{N}\left|\left(\phi^{\star} u\right)(a)\right|\right\}=\sup _{a \in \mathcal{A}}\left\{\left.\left\|\phi^{-1}(a)\right\|\right|^{N}|u(a)|\right\} .
$$

By Proposition 6.1, one can find $r>0$ such that

$$
\sup _{a \in \mathcal{A}}\left\{\left\|\phi^{-1}(a)\right\|^{N}|u(a)|\right\} \leq r \sup _{a \in \mathcal{A}}\left\{\|a\|^{N}|u(a)|\right\} .
$$

Now, consider $A \in \mathcal{A}$ and let

$$
D_{A}\left(\phi^{\star} u\right)(a)=\left.\frac{d}{d t}\right|_{0} \phi^{\star} u(a+t A) .
$$

In order to bound $\sup _{a \in \mathcal{A}}\left\{\|a\|^{N}\left|\left(D_{A}\left(\phi^{\star} u\right)\right)(a)\right|\right\}$, the preceding argument leads us to look at

$$
\left\langle\phi_{\star \phi^{-1}(a)}(A),\left.d u\right|_{a}\right\rangle,
$$

that is, at $\left\|\phi_{\star \phi^{-1}(a)}(A)\right\|$. Since

$$
\left.\frac{d}{d t}\right|_{0} \Omega\left(\phi\left(\phi^{-1}(a)+t A\right), l\right)=\xi\left(\cosh \left(\phi^{-1}(a)\right) \rho(A) l\right)
$$

(using the definition of $\phi$ ), one only needs to analyze the asymptotic behavior of $\cosh \left(\phi^{-1}(a)\right)$. For this, observe that for some $m>0$ and for all $a \in \mathcal{A}$, one has:

$$
\begin{aligned}
\|a\|^{-m}\left\|\left(\cosh \left(\phi^{-1}(a)\right)\right)^{-1}\right\| & \left.=\left\|\phi\left(a^{\prime}\right)\right\|^{-m} \|\left(\cosh \left(a^{\prime}\right)\right)\right)^{-1} \| \\
& \left.\leq\left\|a^{\prime}\right\|^{-m} \|\left(\cosh \left(a^{\prime}\right)\right)\right)^{-1} \|,
\end{aligned}
$$

as soon as $\left\|a^{\prime}=\phi^{-1}(a)\right\|$ is large enough. Therefore, using Lemma 6.6, there exists $m>0$ such that

$$
\|\| a\left\|^{-m}\left(\cosh \left(\phi^{-1}(a)\right)\right)^{-1}\right\| \leq 1
$$

for $\|a\|$ large enough. Hence, Lemma 6.8 implies:

$$
\|a\|^{2 m}\left\|\left(\cosh \left(\phi^{-1}(a)\right)\right)^{2}\right\| \geq\|a\|^{m}\left\|\cosh \left(\phi^{-1}(a)\right)\right\|
$$

that is, by Lemma 6.7:

$$
\left\|\cosh \left(\phi^{-1}(a)\right)\right\| \leq\|a\|^{m}\left\|\mathbf{1}+(\rho(a))^{2}\right\|
$$


which has a polynomial growth.

Now, we indicate how to prove (i) by induction over the order of derivation. Let $\nabla$ be the flat Euclidean connection on the vector space $\mathcal{A}$. For $u \in C^{\infty}(\mathcal{A})$, let $\nabla^{(r)} u$ be the symmetrization of the tensor field $\nabla^{r} u$. For the two first orders of derivation, one then has

$$
D_{A}\left(\phi^{\star} u\right)=\left\langle\nabla u \circ \phi,\left(\phi_{\star}(A)\right) \circ \phi\right\rangle=\phi^{\star}\left\langle\nabla u, \phi_{\star} A\right\rangle
$$

and

$$
D_{B} D_{A}\left(\phi^{\star} u\right)=\phi^{\star}\left(\left\langle\left\langle\nabla^{(2)} u, \phi_{\star} B\right\rangle, \phi_{\star} A\right\rangle+\left\langle\nabla u,\left\langle\nabla\left(\phi_{\star} A\right), \phi_{\star} B\right\rangle\right\rangle\right) .
$$

The functions (sections) $\nabla u$ and $\nabla^{(2)} u$ are Schwartz and we have seen that $\phi_{\star} A$ has polynomial growth. So, in order to bound the second derivative $D_{B} D_{A}\left(\phi^{\star} u\right)$, one needs to control the asymptotic behavior of $\nabla\left(\phi_{\star} A\right)$, that is $\Omega\left(\left\langle\nabla\left(\phi_{\star} A\right), B\right\rangle, l\right)$ for all $A, B \in \mathcal{A}, l \in \mathcal{L}$. One has

$$
\begin{aligned}
\Omega\left(\left.\left\langle\nabla\left(\phi_{\star} A\right), B\right\rangle\right|_{a}, l\right) & =\left.\frac{d}{d t}\right|_{0} \Omega\left(\phi_{\star_{\phi^{-1}}(a+t B)}(A), l\right) \\
& =\xi\left(\sinh \left(\phi^{-1}(a)\right)\left(\cosh \left(\phi^{-1}(a)\right)\right)^{-1} \rho(A) \rho(B) l\right) \\
& =\xi\left(\rho(a)\left(\cosh \left(\phi^{-1}(a)\right)\right)^{-1} \rho(A) \rho(B) l\right) .
\end{aligned}
$$

We have seen previously that $\left(\cosh \left(\phi^{-1}(a)\right)\right)^{-1}$ has polynomial growth. Therefore, $\nabla\left(\phi_{\star} A\right)$ has polynomial growth too. Now, by using the Leibniz identity and an induction argument, one gets (i).

For (ii), we first look for a positive number $N$ such that

$$
\int_{\mathcal{A}_{o}}\|a\|^{-N}\left|\left(\phi^{-1}\right)^{\star} u(a)\right| d a<\infty \quad(u \in \mathcal{S}(\mathcal{A})),
$$

where $\mathcal{A}_{o}$ is the complement of some compact neighborhood of the origin in $\mathcal{A}$. A change of variables following $a \leftarrow \phi(a)$ leads us to

$$
\int_{\mathcal{A}_{o}^{\prime}}\|\phi(a)\|^{-N}\left|\operatorname{Jac}_{\phi}(a)\right||u(a)| d a
$$

where $\operatorname{Jac}_{\phi}(a)$ denotes the determinant of the differential of $\phi$ at point $a$, and where $\mathcal{A}_{o}^{\prime}$ is of the same type as $\mathcal{A}_{o}$ (note that the origin is fixed by the diffeomorphism $\phi$ ). With the notations adopted in the proof of Lemma 6.2, one observes that $\operatorname{Jac}_{\phi}(a)$ is proportional to $\Pi_{\lambda \in \Phi}|\cosh (\lambda(a))|^{\operatorname{dim}\left(V_{\lambda}\right)}$. Therefore, for some constant $c>0$, one has

$$
\begin{aligned}
\left|\operatorname{Jac}_{\phi}(a)\right| & \leq c \max \left\{1,\left(\Pi_{\lambda \in \Phi}|\cosh (\lambda(a))|^{\operatorname{dim}\left(V_{\lambda}\right)}\right)^{2}\right\} \\
& =c \max \left\{1, \Pi_{\lambda \in \Phi}\left|\sinh ^{2}(\lambda(a))+1\right|^{\operatorname{dim}\left(V_{\lambda}\right)}\right\} .
\end{aligned}
$$


Thus

$$
\begin{aligned}
\left|\operatorname{Jac}_{\phi}(a)\right| & \leq c \max \left\{1, \Pi_{\lambda \in \Phi}\left(\left|\sinh ^{2}(\lambda(a))\right|+1\right)^{\operatorname{dim}\left(V_{\lambda}\right)}\right\} \\
& \leq c\left(1+\|\sinh (a)\|^{2}\right)^{K}=c\left(1+\|\phi(a)\|^{2}\right)^{K}
\end{aligned}
$$

for some $K>0$ (cf. proof of Proposition 6.1). This last expression being lower than $\|\phi(a)\|^{N}$ for some $N>0$ and $\|a\|$ large enough, this provides the desired $N$ in order to bound (15) hence (14).

For derivatives of $\left(\phi^{-1}\right)^{\star} u$, an argument as in (i) leads us to consider $\phi_{\star \phi(a)}^{-1}(A) \quad(a, A \in \mathcal{A})$ that is to consider the inverse matrix $\left[\phi_{\star a}\right]^{-1}$ i.e., $\cosh (a)^{-1}$. Hence, by use of Lemma 6.6 , one now gets

$$
\int_{\mathcal{A}_{o}} \frac{1}{\|a\|^{N}}\left|\left(D_{A}\left(\phi^{-1}\right)^{\star} u\right)(a)\right| d a<\infty \quad \forall A \in \mathcal{A} ; u \in \mathcal{S}(\mathcal{A}) .
$$

Similarly to (i), an induction yields (ii).

Set $\overline{\mathcal{P}}=\mathcal{A} \times \mathcal{A}$. Identifying $\mathcal{L}^{\star}$ with $\mathcal{A}$ via the symplectic structure $\Omega$, one can consider the partial Fourier transform:

$$
\mathcal{S}^{\prime}(\mathcal{P}) \stackrel{F}{\rightarrow} \mathcal{S}^{\prime}(\overline{\mathcal{P}})
$$

formally given by

$$
F u(a, \alpha)=\hat{u}(a, \alpha)=\int_{\mathcal{L}} e^{-i \Omega(\alpha, l)} u(a, l) d l .
$$

One denotes its inverse by $\mathcal{S}^{\prime}(\mathcal{P}) \stackrel{F^{-1}}{\rightarrow} \mathcal{S}^{\prime}(\overline{\mathcal{P}})$.

Now, led by Section 5 , we make the following definition.

Definition 6.9. For all $\hbar>0$, we denote by $\varphi_{\hbar}: \overline{\mathcal{P}} \rightarrow \overline{\mathcal{P}}$ the diffeomorphism defined by

$$
\varphi_{\hbar}(a, \alpha)=\left(a, \frac{2}{\hbar} \phi\left(\frac{\hbar}{2} \alpha\right)\right) .
$$

We denote by $\mathcal{S}(\mathcal{P}) \stackrel{\tau_{\hbar}}{\rightarrow} \mathcal{S}^{\prime}(\mathcal{P})$ the map

$$
\tau_{\hbar}=F^{-1} \circ \varphi_{\hbar}^{-1^{\star}} \circ F \text {. }
$$

We set

$$
\mathcal{E}_{\hbar} \stackrel{\text { def. }}{=} \tau_{\hbar}(\mathcal{S}(\mathcal{P})) \subset \mathcal{S}^{\prime}(\mathcal{P}),
$$

and define a map $\mathcal{E}_{\hbar} \stackrel{T_{\hbar}}{\rightarrow} \mathcal{S}(\mathcal{P})$ by

$$
T_{\hbar}=F^{-1} \circ \varphi_{\hbar}^{\star} \circ F
$$


Remark 6.10. In the nilpotent case (i.e., $\mathcal{G}$ nilpotent), one has $\mathcal{E}_{\hbar}=\mathcal{S}(\mathcal{P})$.

\section{Proposition 6.11.}

(i) $\mathcal{S}(\mathcal{P}) \subset \mathcal{E}_{\hbar}$.

(ii) $T_{\hbar} \circ \tau_{\hbar}=i d_{\mathcal{S}(\mathcal{P})}$.

(iii) $\left.\tau_{\hbar} \circ T_{\hbar}\right|_{\mathcal{S}(\mathcal{P})}=i d_{\mathcal{S}(\mathcal{P})}$.

(iv) Let $\star_{\hbar}^{0}$ be the Weyl product on $\mathcal{S}(\mathcal{P})$ (see formula (1) in Section 1). Then, the expression

$$
a \star_{\hbar} b \stackrel{\text { def. }}{=} \tau_{\hbar}\left(T_{\hbar} a \star_{\hbar}^{0} T_{\hbar} b\right) \quad a, b \in \mathcal{E}_{\hbar}
$$

defines an associative algebra structure on $\mathcal{E}_{\hbar}$.

Proof. By Proposition 6.5, for all $u \in \mathcal{S}(\mathcal{P})$, one has $\left(F^{-1} \circ \varphi_{\hbar}^{\star} \circ F\right) u \in \mathcal{S}(\mathcal{P})$. Hence $\tau_{\hbar}\left(F^{-1} \circ \varphi_{\hbar}^{\star} \circ F\right) u=u \in \tau_{\hbar}(\mathcal{S}(\mathcal{P}))=\mathcal{E}_{\hbar}$. Items (i), (ii) and (iii) follow. Associativity of the Weyl product on $\mathcal{S}(\mathcal{P})$ yields (iv).

Notations 6.12. Since $\Omega\left(\frac{2}{\hbar} \phi\left(\frac{\hbar}{2} \alpha\right), l\right)=\frac{2}{\hbar} \xi\left(\sinh \left(\frac{\hbar}{2} \alpha\right) l\right)$, by setting $\varphi_{0}=i d_{\overline{\mathcal{P}}}$ and $T_{0}=i d_{\mathcal{S}(\mathcal{P})}$, one gets a (separately continuous) map $[0, \infty) \times \mathcal{S}(\mathcal{P}) \rightarrow$ $\mathcal{S}(\mathcal{P}):(\hbar, u) \rightarrow T_{\hbar}(u)$. Furthermore, a computation shows that $d_{\lambda} \circ F=$ $\frac{1}{\lambda^{n}} F \circ d_{\lambda}$, where $d_{\lambda}$ is defined as follows:

$$
d_{\lambda}: C^{\infty}(\mathcal{P}) \rightarrow C^{\infty}(\mathcal{P}):\left(d_{\lambda} u\right)(a, l)=u(a, \lambda l) \text { for } \lambda \in \mathbb{R}_{0} .
$$

Therefore, one has

$$
T_{\hbar}=d_{\frac{2}{\hbar}} \circ T_{2} \circ d_{\frac{\hbar}{2}} \quad(\hbar>0) .
$$

In particular, $\mathcal{E}_{\hbar}=d_{\frac{2}{\hbar}}(\mathcal{E})$, with $\mathcal{E}=\tau_{2}\left(\mathcal{S}(\mathcal{P})\right.$ ). This leads to set $\mathcal{E}_{0}=\mathcal{S}(\mathcal{P})$ and $u \star_{0} v=u v \quad \forall u, v \in \mathcal{S}(\mathcal{P})$.

Theorem 6.13. Let $(M, \omega, s)$ be an elementary solvable symplectic symmetric space admitting a globally defined midpoint map. Let $G$ be its transvection group. Let $\phi: \mathcal{A} \rightarrow \mathcal{A}$ be the twisting diffeomorphism defined in Proposition 4.4. Then, the family $\left\{\mathcal{E}_{\hbar}\right\}_{\hbar \geq 0}$ (cf. Definition 6.9), defines a $G$-invariant WKB-quantization of $(M, \omega, s)$ (cf. Definition 1.5). More precisely, let $S \in C^{\infty}(M \times M \times M, \mathbb{R})$ be defined by

$$
S\left(\left(a_{1}, l_{1}\right),\left(a_{2}, l_{2}\right),\left(a_{3}, l_{3}\right)\right)=\xi\left(\oint_{1,2,3} \sinh \left(a_{1}-a_{2}\right) l_{3}\right)
$$


(cf. Proposition 4.9). Then, for all $u$ and $v$ in $\mathcal{D}(M) \subset \mathcal{E}_{\hbar}$, the product reads:

$$
\begin{aligned}
& u \star \hbar v(x) \\
& =\frac{1}{\hbar^{2 n}} \int_{M \times M} e^{\frac{2 i}{\hbar} S\left(x, x_{1}, x_{2}\right)}\left|\operatorname{det}\left(\left.\cosh \left(a_{2}-a_{1}\right)\right|_{\mathcal{L}}\right)\right| u\left(x_{1}\right) v\left(x_{2}\right) d x_{1} d x_{2}
\end{aligned}
$$

with $x_{i}=\left(a_{i}, l_{i}\right) \in M=\mathcal{P}(i=1,2)$, and where $d x$ stands for the symplectic measure on $M=\mathcal{P}$. The phase function $S$ as well as the amplitude $\left|\operatorname{det}\left(\left.\cosh \left(a_{2}-a_{1}\right)\right|_{\mathcal{L}}\right)\right|$ are invariant under the symmetries $\left\{s_{x}\right\}_{x \in M}$.

Proof. For the sake of simplicity, we establish the product formula (16) for $\hbar=2$. We set $\star^{0}=\star_{2}^{0}$ (Weyl's product) $T=T_{2}, \tau=\tau_{2}$ and $\varphi=\varphi_{2}$. Also, in the formulae that follow, integration is taken over every variable $a_{i}, l_{i}, \alpha_{i}$ with $i=1,2$. Let $u, v \in \mathcal{D}(M)$ be compactly supported. Then, formally, one has:

$$
\begin{aligned}
& \left(T u \star^{0} T v\right)\left(a_{0}, l_{0}\right) \\
& =\int e^{i S^{0}\left(\left(a_{0}, l_{0}\right),\left(a_{1}, l_{1}\right),\left(a_{2}, l_{2}\right)\right)} e^{i \Omega\left(\alpha_{1}, l_{1}\right)}\left(\varphi^{\star} \hat{u}\right)\left(a_{1}, \alpha_{1}\right) e^{i \Omega\left(\alpha_{2}, l_{2}\right)}\left(\varphi^{\star} \hat{v}\right)\left(a_{2}, \alpha_{2}\right) \\
& =\int e^{i\left[\Omega\left(a_{2}-a_{1}, l_{0}\right)+\Omega\left(a_{0}-a_{2}+\alpha_{1}, l_{1}\right)+\Omega\left(a_{1}-a_{0}+\alpha_{2}, l_{2}\right)\right]}\left(\varphi^{\star} \hat{u}\right)\left(a_{1}, \alpha_{1}\right)\left(\varphi^{\star} \hat{v}\right)\left(a_{2}, \alpha_{2}\right)
\end{aligned}
$$

(using the definition of the Weyl product)

$$
\begin{aligned}
=\int e^{i \Omega\left(a_{2}-a_{1}, l_{0}\right)}\left[\int e^{i \Omega\left(a_{0}-a_{2}+\alpha_{1}, l_{1}\right)}\left(\varphi^{\star} \hat{u}\right)\left(a_{1}, \alpha_{1}\right)\right. \\
\left.\cdot \int e^{i \Omega\left(a_{1}-a_{0}+\alpha_{2}, l_{2}\right)}\left(\varphi^{\star} \hat{v}\right)\left(a_{2}, \alpha_{2}\right)\right] \\
=\int e^{i \Omega\left(a_{2}-a_{1}, l_{0}\right)}\left(\varphi^{\star} \hat{u}\right)\left(a_{1}, a_{2}-a_{0}\right)\left(\varphi^{\star} \hat{v}\right)\left(a_{2}, a_{0}-a_{1}\right) .
\end{aligned}
$$

Moreover,

$$
\begin{aligned}
\tau u(a, l) & =\int e^{i \Omega(\alpha, l)}\left(\varphi^{-1^{\star}} \hat{u}\right)(a, \alpha) d \alpha \\
& =\int e^{i \Omega(\alpha, l)} \hat{u}\left(a, \phi^{-1}(\alpha)\right) d \alpha \\
& =\int e^{i\left(\Omega(\alpha, l)-\Omega\left(\phi^{-1}(\alpha), \lambda\right)\right)} u(a, \lambda) d \alpha d \lambda .
\end{aligned}
$$


Hence

$$
\begin{aligned}
\tau & \left(T u \star^{0} T v\right)\left(a_{0}, l_{0}\right) \\
= & \int e^{i\left(\Omega\left(\alpha, l_{0}\right)-\Omega\left(\phi^{-1}(\alpha), \lambda\right)\right)} e^{i \Omega\left(a_{2}-a_{1}, \lambda\right)} \\
& \cdot\left(\varphi^{\star} \hat{u}\right)\left(a_{1}, a_{2}-a_{0}\right)\left(\varphi^{\star} \hat{v}\right)\left(a_{2}, a_{0}-a_{1}\right) d \lambda d \alpha \\
= & \int e^{i\left[\Omega\left(\alpha, l_{0}\right)-\Omega\left(\phi^{-1}(\alpha), \lambda\right)+\Omega\left(a_{2}-a_{1}, \lambda\right)-\Omega\left(\phi\left(a_{2}-a_{0}\right), l_{1}\right)-\Omega\left(\phi\left(a_{0}-a_{1}\right), l_{2}\right)\right]} \\
& \cdot u\left(a_{1}, l_{1}\right) v\left(a_{2}, l_{2}\right) d \lambda d \alpha \\
= & \int e^{i\left[\Omega\left(\phi(\alpha), l_{0}\right)-\Omega(\alpha, \lambda)+\Omega\left(a_{2}-a_{1}, \lambda\right)-\Omega\left(\phi\left(a_{2}-a_{0}\right), l_{1}\right)-\Omega\left(\phi\left(a_{0}-a_{1}\right), l_{2}\right)\right]} \\
& \cdot\left|J a c_{\phi}(\alpha)\right| u\left(a_{1}, l_{1}\right) v\left(a_{2}, l_{2}\right) d \lambda d \alpha
\end{aligned}
$$

(after changing the variables following $\alpha \leftarrow \phi(\alpha)$ )

$$
\begin{aligned}
= & \int e^{i\left[\Omega\left(\phi(\alpha), l_{0}\right)-\Omega\left(\phi\left(a_{2}-a_{0}\right), l_{1}\right)-\Omega\left(\phi\left(a_{0}-a_{1}\right), l_{2}\right)-\Omega\left(\alpha-a_{2}+a_{1}, \lambda\right)\right]} \\
& \cdot\left|J a c_{\phi}(\alpha)\right| u\left(a_{1}, l_{1}\right) v\left(a_{2}, l_{2}\right) d \lambda d \alpha \\
= & \int e^{i\left[\Omega\left(\phi\left(a_{2}-a_{1}\right), l_{0}\right)-\Omega\left(\phi\left(a_{2}-a_{0}\right), l_{1}\right)-\Omega\left(\phi\left(a_{0}-a_{1}\right), l_{2}\right)\right]} \\
& \cdot\left|J a c_{\phi}\left(a_{2}-a_{1}\right)\right| u\left(a_{1}, l_{1}\right) v\left(a_{2}, l_{2}\right) \\
= & \int e^{i S\left(x_{0}, x_{1}, x_{2}\right)}\left|J a c_{\phi}\left(a_{2}-a_{1}\right)\right| u\left(x_{1}\right) v\left(x_{2}\right)
\end{aligned}
$$

(using the definition of $\phi$ ). We now get the announced formula using item (ii) of Remark 4.5. Dirac's condition is implied by Fedoriuk's formula (formula (1.5) p. 30 in [15]) up to order one in the parameter $\hbar$, provided $(u \star \hbar v)(x)$ is interpreted as the oscillatory integral:

$$
\int \varphi(X) \exp \left(\frac{i}{\hbar} \Sigma(X)\right) d X
$$

with

$$
\begin{aligned}
& X=\left(x_{1}, x_{2}\right) \in M \times M, \\
& \varphi(X)=\left|\operatorname{det}\left(\left.\cosh \left(a_{2}-a_{1}\right)\right|_{\mathcal{L}}\right)\right| u \otimes v(X) \text { and } \\
& \Sigma(X)=2 S(x, X) .
\end{aligned}
$$

The critical point analysis of the function $\Sigma$ then tells us that $\Sigma$ has an isolated critical point at $X^{0}=(x, x)$. Moreover, the Hessian matrix $\partial_{X}^{2} \Sigma\left(X^{0}\right)$ is proportional to

$$
\left(\begin{array}{ll}
0 & \Omega \\
\Omega & 0
\end{array}\right)
$$


on $T_{X^{0}}(M \times M)=\mathcal{P} \times \mathcal{P}$. Hence $\left(u \star_{\hbar} v\right)(x)$ admits the desired asymptotic expansion.

\section{Topological algebras.}

In this section, we analyze some topological properties of the algebras $\mathcal{E}_{\hbar} \quad(\hbar>0)$ defined in Section 6. Each of these algebras being isomorphic, via a "dilation", to the algebra $\mathcal{E}=\tau_{2}(\mathcal{S}(\mathcal{P})$ ) (cf. Notations 6.12), we will, in this section, drop the symbol " $\hbar$ " in our discussion. As previously, we set $\tau=\tau_{2}, T=T_{2}$ and $\star=\star_{2}$. Moreover, we set $\mathcal{S}=\mathcal{S}(\mathcal{P})$ and $\mathcal{D}=\mathcal{D}(M)$.

Definition 7.1. On $\mathcal{S}$, let us denote by $(,)_{\mathcal{S}}$ the canonical $L^{2}$-inner product

$$
(u, v)_{\mathcal{S}}=\int u \bar{v}
$$

Via the linear bijection $T: \mathcal{E} \rightarrow \mathcal{S}$, one define on $\mathcal{E}$ the following inner product

$$
(a, b)_{\mathcal{E}} \stackrel{\text { def. }}{=}(T a, T b)_{\mathcal{S}}
$$

The space $\mathcal{E}$ then becomes a pre-Hilbert space whose Hilbert completion is denoted by $\mathcal{H}$.

\section{Proposition 7.2.}

(i) The inclusions

$$
\mathcal{D} \subset \mathcal{S} \subset \mathcal{E} \subset \mathcal{H}
$$

are dense.

(ii) For all $x \in M$ and $u, v \in \mathcal{D}$, one has

$$
\left(s_{x}^{\star} u, s_{x}^{\star} v\right)_{\mathcal{E}}=(u, v)_{\mathcal{E}} .
$$

In particular, the action of the transvection group $G$ on $\mathcal{D}$ extends as an (unitary) action of $G$ on $\mathcal{H}$.

(iii) The algebra structure $\star$ on $\mathcal{E}$ extends to $\mathcal{H}$. Endowed with the extended product again denoted by $\star$, the space $\mathcal{H}$ becomes an associative topological algebra.

(iv) The group $G$ acts on $(\mathcal{H}, \star)$ by algebra automorphisms. 
Proof. Observe that the space $F^{-1} \mathcal{D}$ is stable by both transformations $T$ and $\tau$. Moreover, the inclusion $F^{-1} \mathcal{D} \subset \mathcal{S}$ is dense with respect to the $L^{2}$ topology. Hence $\tau F^{-1} \mathcal{D}=F^{-1} \mathcal{D}$ is dense in $\mathcal{E}$ and so is $\mathcal{S}$. Moreover, the $\left.\operatorname{map} T\right|_{\mathcal{S}}: \mathcal{S} \rightarrow \mathcal{S}$ is continuous with respect to the $L^{2}$-topology. Indeed, this follows from the fact that

$$
\begin{aligned}
\left|\operatorname{Jac}_{\phi^{-1}}(a)\right| & =\frac{1}{\left|\operatorname{det}\left(\phi_{\star_{\phi^{-1}}(a)}\right)\right|} \\
& =\frac{1}{\left|\operatorname{det}\left(\left.\cosh \left(\phi^{-1}(a)\right)\right|_{\mathcal{L}}\right)\right|} \\
& =\frac{1}{\left|\operatorname{det}\left(\left.\cosh \left(\phi^{-1}(a)\right)\right|_{\mathcal{L}}\right)^{2}\right|^{\frac{1}{2}}} \\
& =\frac{1}{\left|\operatorname{det}\left(\mathbf{1}+\left.\left(\rho(a)^{2}\right)\right|_{\mathcal{L}}\right)\right|^{\frac{1}{2}}} .
\end{aligned}
$$

Hence the inclusion $\mathcal{S} \stackrel{i}{\rightarrow} \mathcal{E}, i=\left.\tau \circ T\right|_{\mathcal{S}}$, is continuous when $\mathcal{S}$ is endowed with the $L^{2}$-topology. This yields (i).

One has, with $x=\left(a_{0}, l_{0}\right)$ :

$$
\begin{aligned}
\left(s_{x}^{\star} u, s_{x}^{\star} v\right)_{\mathcal{E}}= & \left(F^{-1} \varphi^{\star} F s_{x}^{\star} u, F^{-1} \varphi^{\star} F s_{x}^{\star} v\right)_{\mathcal{S}(\mathcal{P})} \\
= & \left(\varphi^{\star} F s_{x}^{\star} u, \varphi^{\star} F s_{x}^{\star} v\right)_{\mathcal{S}(\overline{\mathcal{P}})} \\
= & \int\left|J a c_{\phi^{-1}}\right| F s_{x}^{\star} u \overline{F s_{x}^{\star} v} \\
= & \int\left[\left|J a c_{\phi^{-1}}(\alpha)\right| \int e^{i \Omega(\alpha, l)} u\left(2 a_{0}-a, 2 \cosh \left(a_{0}-a\right) l_{0}-l\right) d l\right. \\
& \left.\cdot \int^{-i \Omega\left(\alpha, l^{\prime}\right)} \bar{v}\left(2 a_{0}-a, 2 \cosh \left(a_{0}-a\right) l_{0}-l^{\prime}\right) d l^{\prime}\right] d a d \alpha \\
= & \int\left[\left|J a c_{\phi^{-1}}(\alpha)\right| \int e^{-i \Omega\left(\alpha, \lambda-2 \cosh \left(a_{0}-a\right) l_{0}\right)} u\left(2 a_{0}-a, \lambda\right) d \lambda\right. \\
& \left.\cdot \int e^{i \Omega\left(\alpha, \lambda^{\prime}-2 \cosh \left(a_{0}-a\right) l_{0}\right)} \bar{v}\left(2 a_{0}-a, \lambda^{\prime}\right) d \lambda^{\prime}\right] d a d \alpha .
\end{aligned}
$$

Since the terms $\Omega\left(\alpha, 2 \cosh \left(a_{0}-a\right) l_{0}\right)$ in the exponentials cancel each other, the latter expression equals

$$
\int d a d \alpha\left|J a c_{\phi^{-1}}(\alpha)\right| \overline{F \bar{u}}\left(2 a_{0}-a, \alpha\right) F \bar{v}\left(2 a_{0}-a, \alpha\right)=(u, v)_{\mathcal{E}}
$$


This proves (ii) while item (iii) follows from [11]. Now, observe that for $a=\lim a_{n} \in \mathcal{H}$ with $a_{n} \in \mathcal{D}, u \in \mathcal{D}$ and $g \in G$, one has

$$
\begin{aligned}
& g a \star g u=\left(\lim g a_{n}\right) \star g u \quad \text { (by (ii)) } \\
& =\lim \left(g a_{n} \star g u\right) \quad \text { (by (iii)) } \\
& =\lim g\left(a_{n} \star u\right) \quad \text { (by formula (16)) } \\
& =g \lim \left(a_{n} \star u\right) \quad \text { (by (ii)) } \\
& =g(a \star u) \quad \text { (by (iii)). }
\end{aligned}
$$

This implies (iv).

We now follow a standard procedure ([11], [21]). For all $a \in \mathcal{H}$, the left multiplication $L_{a}: \mathcal{H} \rightarrow \mathcal{H}: b \rightarrow a \star b$ is a bounded operator. This yields an algebra homomorphism

$$
\mathcal{H} \rightarrow \mathcal{B}(\mathcal{H}): a \rightarrow L_{a}
$$

into the $C^{\star}$-algebra $\mathcal{B}(\mathcal{H})$ of bounded operators on $\mathcal{H}$. This homomorphism is continuous. It is also injective. Indeed, denoting Weyl's product by $\star^{0}$, our Hilbert algebra $(\mathcal{H}, \star)$ is isomorphic to $\left(L^{2}(\mathcal{P}), \star^{0}\right)$. Denoting by $\operatorname{tr}^{0}$ the canonical trace for Weyl's quantization (i.e $\operatorname{tr}^{0}(u)=\int u$ ), one has

$$
\operatorname{tr}^{0}\left(a \star^{0} \bar{b}\right)=(a, b)_{L^{2}} \quad \forall a, b \in L^{2}(\mathcal{P}) .
$$

Hence, for $a \in \mathcal{H}$ such that $L_{a}=0$, one gets $(a, \mathcal{H})=0$, that is $a=0$. One therefore gets a new norm on $\mathcal{H}$ :

$$
\|a\|_{2} \stackrel{\text { def. }}{=}\left\|L_{a}\right\|_{\mathcal{B}(\mathcal{H})} \text {. }
$$

\section{Proposition 7.3.}

(i) The complex conjugation on $\mathcal{D}$ extends continuously to the Hilbert algebra $\mathcal{H}$ as an involution for the product $\star$. We denote this involution by $a \rightarrow a^{*}$.

(ii) The quadruple $\left(\mathcal{H}, \star,\|\|_{2},{ }^{*}\right)$ is then a (pre)-C $C^{*}$-algebra on which the transvection group acts by $C^{*}$-algebra automorphisms.

Proof. Let $u \in \mathcal{D}$ and note that, from $\varphi^{\star} F \circ F(u)=F \circ F\left(\varphi^{\star} u\right)$, one gets $T(\bar{u})=\overline{T(u)}$. Therefore, one obtains the pre- $C^{\star}$-algebra structure on $(\mathcal{H}, \star)$ by transporting the one on $\left(L^{2}(\mathcal{P}), \star^{0}\right)[11]$ via the isomorphism

$$
T: \mathcal{H} \rightarrow\left(L^{2}(\mathcal{P})\right. \text {. }
$$

The rest follows from Proposition 7.2. 


\section{Remarks for further developments.}

A possible extension of this work is to define curved "quantum symmetric solv-manifolds" analogue to flat quantum tori. That is, strict quantizations (in Rieffel's sense) of compact quotients of solvable symmetric spaces. The presence of curvature should yield interesting continuous fields of $C^{\star}$ algebras. Indeed, already at the level of the universal covering, which is considered in this present work, one can observe the non closeness of the Schwartz space $\mathcal{S}$ under our deformed products: the one parameter "equivalence" $T_{\hbar}$ really "turns" $\mathcal{S}$ inside $C_{\infty}(M)$ (cf. Section 6). It is not even clear if there actually exists any reasonable Poisson subspace stable, through the deformation, under both classical and deformed products. In order to attend these questions, one would need to focuse the following points.

1. Establish continuous fields. That is, firstly, describe the strong deformation of $\mathcal{S}(\hbar=0)$ with generic fiber $\mathcal{H}$ (cf. Proposition 7.3) arising from our construction. Secondly, extend this to (smooth) bounded functions - this is necessary in order to consider quotients later on. Observe that, regarding this last point, the proofs of Proposition 6.1 and Proposition 6.5 indicate that our setting should extend to bounded functions without difficulty just as in the flat case (cf. [21]).

2. Extend the present work to the whole class of solvable symplectic symmetric spaces which admit globally defined midpoint maps. Proposition 3.2 in [7] indicates that this question should follow from an induction on the successive split extensions by Abelian algebras which eventually yields any solvable symmetric space from the data of an elementary one.

3. Study cocompact actions of discrete subgroups of automorphism groups of solvable symmetric spaces.

In this context of solv-manifolds, one can then hope to investigate the problem of defining a quantum analogue to the Anosov property for classical flows.

The approach to quantization of symmetric spaces is also aiming at attending quantum Riemann surfaces in a "universal" setting, that is without referring to particular Hilbert space representations. This problem lies in the semisimple world which, from the geometric point of view as well as from the point of view of star representation theory, is more complicated than the solvable situation considered here. This problem has actually 
been investigated by Berezin in [3]. Since then, numerous of important works have emerged concerning this question (see e.g., [19], [20] and [24]) in the framework of Berezin-Toeplitz quantization. Comparisons between the WKB-quantization approach and Berezin's quantization of the hyperbolic plane have first been investigated by Weinstein and Qian (see [25] and [18]). Explicit computations of invariant admissible phase functions on the hyperbolic plane have been performed by Weinstein, Qian and the author. Also, some of the steps of the present construction pass to the case of the hyperbolic plane. For instance explicit Darboux charts in which the Moyal star product is $S L_{2}(\mathbb{R})$-covariant have been found by the author. But, it is unclear how to define an intertwiner analogous to operator $\mathcal{Z}_{\hbar}$ (cf. Section 5) from the data of the star representation cocycle and the twisting map (cf. Definition 4.3) in the case of the hyperbolic plane.

It seems also interesting to compare our framework with Fedosov's invariant quantization. At the formal level, Fedosov's quantization gives us a way to construct invariant star products on affine symplectic manifolds ("invariant" means that the star product is preserved under the affine symplectic transformations) [9]. In the case of a symplectic symmetric space, such an invariant $\star$-product is essentially unique (see [4]). The present work therefore deals with the problem of finding, in our framework of solvable symmetric spaces, "oscillatory integral formulae" whose expansions are Fedosov's series.

At last, in [24], Unterberger and Upmeier study a pseudo-differential calculus (called Fuchs calculus) on symmetric cones. On a given symmetric cone $C$, Fuchs calculus is equivariant under the action of a solvable Lie group $G_{c}$ which is the contraction of the automorphism group of the complex tube domain $\Pi$ over $C$. Explicit integral formulae for the composition products of Fuchs symbols have been obtained in [23]. In some cases, our framework overlaps Unterberger's one and, in such cases, the formulae we obtain for the WKB-quantizations coincide, up to some diffeomorphism which can be interpreted in geometric terms, with Unterberger's composition formulae. Despite the fact that, in our case, no symbol-operator correspondence is used, our work therefore appears to be closely related to Fuchs calculus. A deeper study of the relation with Fuchs calculus will be investigated in a forthcoming paper [8]. 


\section{References.}

[1] D. Arnal and J.-C. Cortet, *-products in the method of orbits for nilpotent groups, J. Geom. Phys., 2(2) (1985), 83-116.

[2] F. Bayen et al., Deformation theory and quantization. II. Physical applications, Ann. Physics, 111(1) (1978), 111-151.

F. Bayen et al., Deformation theory and quantization. I. Deformations of symplectic structures, Ann. Physics, 111(1) (1978), 61-110.

[3] F.A. Berezin, Quantization in complex symmetric spaces, Izv. Akad. Nauk SSSR Ser. Mat., 39(2) (1975), 363-402, 472.

[4] M. Bertelson, P. Bieliavsky and S. Gutt, Parametrizing equivalence classes of invariant star products, Lett. Math. Phys., 46(4) (1998), 339-345.

[5] P. Bieliavsky, Espaces symétriques symlectiques, Ph.D. Thesis, Université Libre de Bruxelles, 1995.

[6] P. Bieliavsky, M. Cahen and S. Gutt, Symmetric symplectic manifolds and deformation quantization, Modern group theoretical methods in Physics, Math. Studies, 18 (1995), 63-75.

[7] P. Bieliavsky, Four-dimensional simply connected symplectic symmetric spaces, Geom. Dedicata, 69(3) (1998), 291-316.

[8] P. Bieliavsky and M. Massar, Strict quantizations for actions of Kählerian Lie groups, in preparation.

[9] B. Fedosov, Deformation quantization and index theory, Akademie Verlag, Berlin, Berlin, 1996.

[10] C. Fronsdal, Some ideas about quantization, Rep. Math. Phys., 15(1) (1979), 111-145.

[11] F. Hanssen, Quantum Mechanics in Phase Space, Reports on Math. Phys. 19(3) (1984), 361-381.

[12] M. Karasev, Formulas for non-commutative products of functions in terms of membranes and strings, I, Russian J. Math. Phys., 2(4) (1994), 445-462. 
[13] S. Kobayashi and K. Nomizu, Foundations of Differential Geometry, Wiley-Interscience, New-York, 1963-1969.

[14] O. Loos, Symmetric Spaces, Benjamin, New-York, 1969.

[15] V.P. Maslov and M.V. Fedoriuk, Semi-Classical Approximation in Quantum Mechanics, Mathematical Physics and Applied Mathematics; 7, D. Reidel Publishing Company, Dordrecht: Holland/ Boston: USA/ London: England, 1981.

[16] G. Nagy, A framework for deformation quantization, Ph.D. Thesis, University of California, Berkeley, 1992.

[17] T. Natsume and R. Nest, Topological approach to quantum surfaces, Commun. Math. Phys., 202(1) (1999), 65-87.

[18] Z. Qian, Groupoids, Midpoints and Quantizations, Ph.D. Thesis, University of California, Berkeley, 1997.

[19] J. Rawnsley, M. Cahen and S. Gutt, Quantization of Kähler manifolds, I. Geometric interpretation of Berezin's quantization, J. Geom. Phys., $\mathbf{7}(\mathbf{1})$ (1990), 45-62;

M. Cahen, S. Gutt and J. Rawnsley, Quantization of Kähler manifolds, IV, Lett. Math. Phys., 34(2) (1995), 159-168.

[20] F. Radulescu, The $\Gamma$-equivariant form of the Berezin quantization of the upper half plane, Mem. Am. Math. Soc., 630 (1998), 70 pp.

[21] M.A. Rieffel, Deformation quantization for actions of $\mathbf{R}^{d}$, Mem. Amer. Math. Soc., 106(506) (1993).

[22] M.A. Rieffel, Deformation Quantization of Heisenberg Manifolds, Commum. Math. Phys., 122 (1989), 531-562.

[23] A. Unterberger, Quantification de certains espaces hermitiens symétriques, Séminaire Goulaouic-Schwartz, 1979-1980 (French), Exp. 16, 13 pp., École Polytech., Palaiseau, 1980.

[24] A. Unterberger and H. Upmeier, Pseudo-differential analysis on symmetric cones, Studies in Advanced Mathematics, 1996. 
[25] A. Weinstein, Traces and triangles in symmetric symplectic spaces, Symplectic geometry and quantization (Sanda and Yokohama, 1993), Contemp. Math., 179 (1994), Amer. Math. Soc., Providence, RI, 261270 .

DÉPARTEMENT DE MATHÉMATIQUes

Université LIBRE DE BRUXELles

CP. 218, Campus Plaine

B-1050, Brussels, Belgium

E-mail address: pbiel@ulb.ac.be 